\title{
Polypores and associated beetles of the North Karelian Biosphere Reserve, eastern Finland
}

\author{
DMITRY S. SCHIGEL, TUOMO NIEMELÄ, MAARIT SIMILÄ, JUHA KINNUNEN and \\ OLLI MANNINEN
}

SCHIGEL, D. S., NIEMELÄ, T., SIMILÄ, M., KINNUNEN, J. \& MANNINEN, O. 2004: Polypores and associated beetles of the North Karelian Biosphere Reserve, eastern Finland. - Karstenia 44: 35-56. Helsinki. ISSN 0453-3402.

Polypores (poroid Basidiomycota) and associated beetles were inventoried in the Koitajoki Natura 2000 area (Ilomantsi municipality) and the Kitsi forest fire area (Lieksa), both belonging to the North Karelian Biosphere Reserve of the EU. As a result 105 polypore species were collected; together with earlier reports by other authors, the amount of species totals 121. This is a high number, surpassed in Finland by a few first-rank nature reserves only. Of the species found, 29 are red listed: 2 endangered (EN: Antrodia crassa (P. Karst.) Ryvarden, Piloporia sajanensis (Parmasto) Niemelä), 11 vulnerable (VU), and 16 near-threatened (NT). Hyphodontia latitans (Bourd. \& Galz.) E. Langer has been found in Finland only once from the same area; now it was recollected. The research area is in a way a meeting-point of some northerly species (e.g. Daedaleopsis septentrionalis (P. Karst.) Niemelä, Trichaptum laricinum (P. Karst.) Ryvarden), those with an eastern distribution in Fennoscandia (e.g. Trichaptum pargamenum (Fr.) G. Cunn.), and some southern ones (e.g. Pycnoporellus fulgens (Fr.) Donk). Remarkably numerous were some species which indicate old growth forests of high conservation value (e.g. Amylocystis lapponica (Romell) Singer, Antrodia albobrunnea (Romell) Ryvarden, A. crassa, Fomitopsis rosea (Alb. \& Schwein. : Fr.) P. Karst., Phellinus nigrolimitatus (Romell) Bourd. \& Galz., Skeletocutis stellae (Pilát) Jean Keller). Beetle imagines were collected from polypore basidiocarps, and their larvae from basidiocarps and underlying decay, and then reared into adults. Special attention was paid to beetles living on rare polypore species. The polypore-associated beetle fauna totals 115 species, including 24 previously unrecorded from the Reserve. Our paper includes beetle records from ca. 30 such polypore species of which no previous beetle finds have been reported in the literature. The ecology of beetles living on fungal basidiocarps is discussed. Polypores can be divided into different ecological groups according to which beetles they attract; a division into basidiocarp consistency classes is proposed to describe such groups. Furthermore, the freshness or decomposition of a basidiocarp determines the amounts of beetles and their larvae, and their species composition.

Key words: Basidiomycota, Coleoptera, Hyphodontia latitans, basidiocarp consistency class, beetles, Finland, polypores, ecology

Dmitry S. Schigel, Tuomo Niemelä, Juha Kinnunen, Olli Manninen, Botanical Museum (Mycology), P. O. Box 7, FI-00014 University of Helsinki, Finland. Correspondence e-mail: dmitry.shchigel@helsinki.fi

Maarit Similä, Finnish Forest and Park Service, Natural Heritage Services, Urheilukatu 3 A, FI-81720 Lieksa, Finland. 


\section{Introduction}

The Koitajoki river and its surroundings in the commune of Ilomantsi, Finnish North Karelia, make up the easternmost Natura 2000 site of the European Union. It is about 7400 ha wide and consists of protection areas with different protection statuses. There are the Koivusuo Strict Nature Reserve, protection areas for peatlands (Ruosmesuo - Hanhisuo and Ristisuo) as well as a reserve for old-growth forests. About 900 ha of the area are under restrictions of the Finnish forest law; there forest management operations are allowed outside of the important key habitats. The remaining 6500 ha belong to the Nature Reserve without forest management.
The Koitajoki Natura 2000 site is an important wilderness area in eastern Finland (Figs. 1, 2). It is situated in the transition zone of northern mires (pohjoiset aapasuot) and southern raised bogs (eteläiset keidassuot). In the area there are relatively large and natural mires and bogs with mosaic-like forests on mineral soil. Most of the forests are seminatural and old, with plenty of dead wood but with some old signs of previous selective cuttings. An important element of this Natura 2000 site is the Koitajoki, a shallow and meandering river with sandy banks (Figs. 3, 4). The Koitajoki Natura 2000 site maintains important rare and threatened species of polypores (Bondarceva et al. 1995; Bondartseva et al. 1998, 1999, 2001, Niemelä et al. 2002), beetles (Yakovlev et al. 2001) and other organisms.

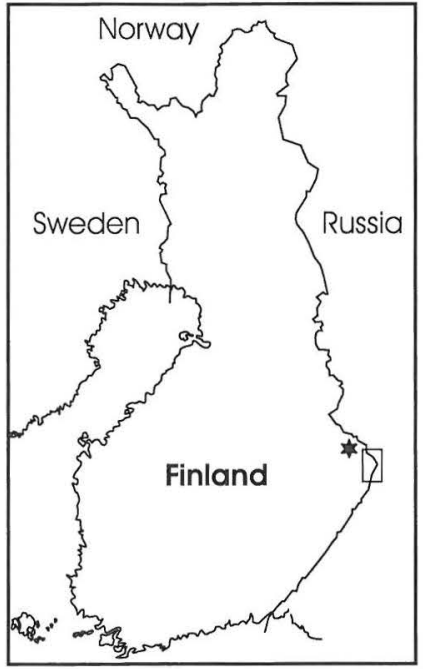

Fig. 1. The research area in easternmost Finland, North Karelian Biosphere Reserve. The Jäkäläkangas Natura 2000 site is marked with an asterisk, rectangular box marks the Koitajoki Natura 2000 site, enlarged on Fig. 2.

Fig. 2. The Koitajoki Natura 2000 site, eastern Finland. Light grey covers the protected area, dark grey the Koivusuo Strict Nature Reserve. Black dots mark the centres of studied plots.

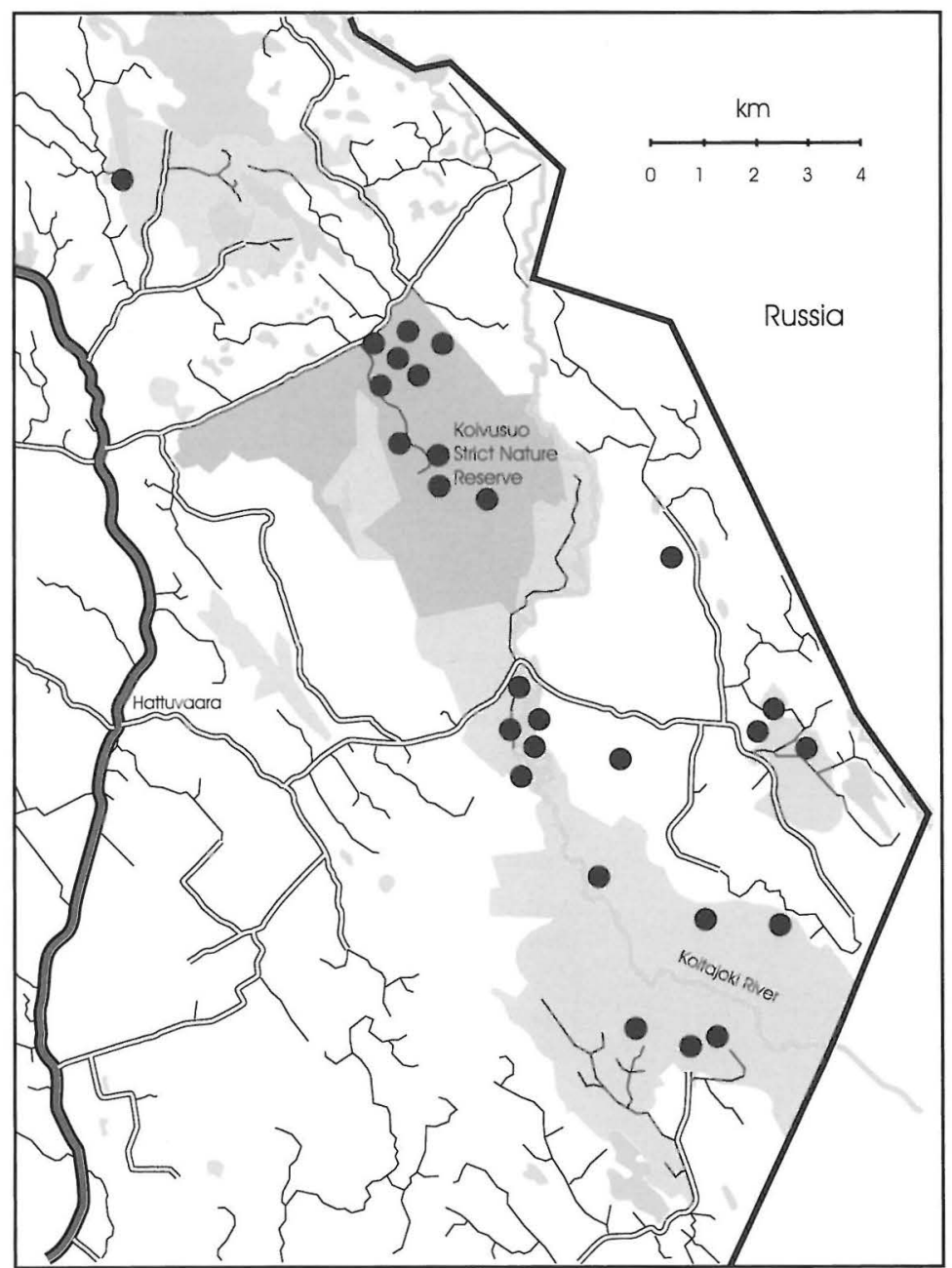


Fig. 3. Meanders of the Koitajoki in the North Karelian Biosphere Reserve near Polvikoski. Photograph TN.

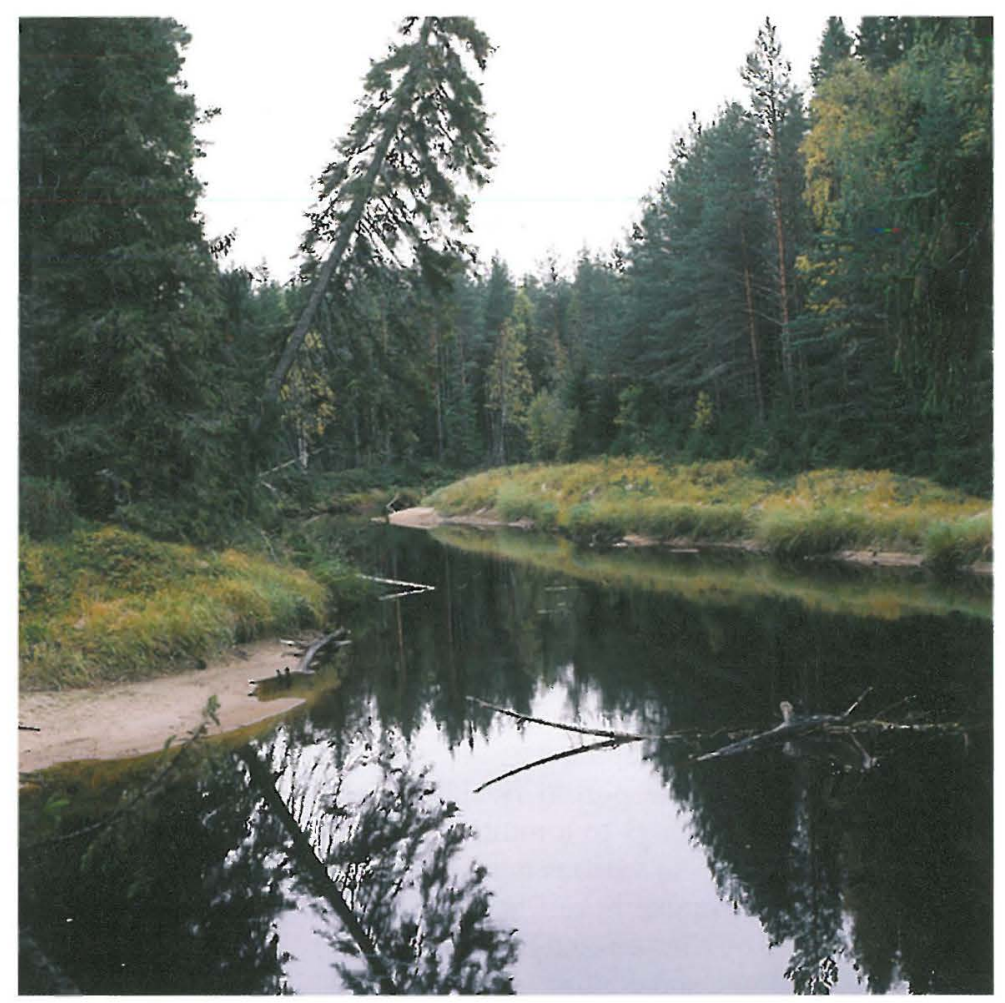

Fig. 4. Old-growth forest in Liekkuaho, the Koitajoki Natura 2000 site. Photograph TN.

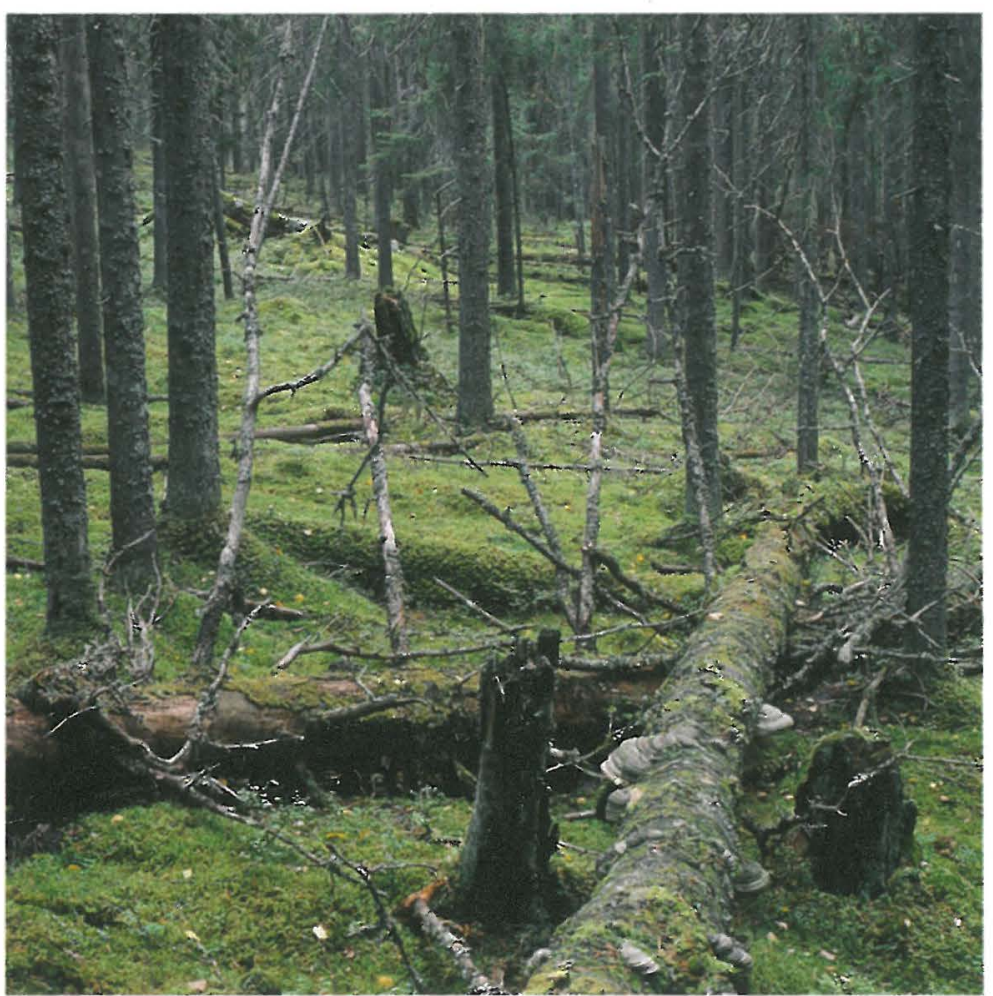


The Jäkäläkangas Natura 2000 site (224 ha) is situated in the municipality of Lieksa. Parts of the Jäkäläkangas area were burned by a wildfire in 1992. So the area includes about 143 ha of naturally burnt forests and peatlands which were protected immediately after the forest fire. About 17 ha were burnt completely to become a very interesting area with plenty of charred dead wood. Polypores were surveyed in the parts of less intensive fire.

Both areas surveyed in September - October 2002 and September 2003 are located inside the North Karelian Biosphere Reserve around Kitsi $\left(63^{\circ} 16^{\prime} \mathrm{N}, 30^{\circ} 44^{\prime} \mathrm{E}\right)$. This first area of the Finnish Biosphere Reserves was established in 1992 (Hokkanen \& Ieshko 1995). Biosphere Reserves are an essential part of UNESCO's Man and Biosphere (MAB) programme. They make up a backbone of the international network of research areas for sustainable development.

The inventory of the poroid Basidiomycete fungi (polypores) belongs to a multi-year study on Finnish polypores in various nature reserves, organized by the Finnish Forest and Park Service. It was started by Niemelä and Dai $(1998,1999)$ in the Luosto range, and continued in Korouoma (Niemelä \& Kinnunen 2001), Ylläs - Aakenus (Niemelä \& Kinnunen 2002) and Pisavaara (Niemelä \& Kinnunen 2003), all of them in northern Finland. This paper is partly based on the 2002 inventory report (Niemelä et al. 2002), but additional collections were made in 2003, and our research materials were further worked out later. Data from the Repovesi National Park, Central Finland (Table 4) derive from an inventory in 2004 (unpublished). Although the polypores of Finland are rather well known, there is still a need in species inventories for better understanding of fungal ecology and nature protection.

European studies in the biology of saproxylic beetles started in the beginning of the 20th century in Scandinavia (Saalas 1917, 1923) and other parts of Europe (Donisthorpe 1931, 1935). From the 1950s the topic was favoured by many coleopterists (e.g. Benick 1952, Palm 1951, 1959, Paviour-Smith 1960). For an overview on Russian saproxylic beetles see Nikitsky and Schigel (2004).

Of more than 30 excellent papers published on the topic from 1990 onwards the most important ones by Økland (1995), Andersen et al. (2000), Thunes et al. (2000), Komonen et al. (2001), Martikainen (2001), Siitonen et al. (2001), Ehnström and Axelsson (2002), Jonsell and Nordlander (2002), describe the fauna and ecology of Fennoscandian saproxylic beetles. Window and trunk beetle trappings (Yakovlev et al. 2001) showed the diversity of the saproxylic beetles of the Reserve. An emphasis of our study was put on search for previously unknown fungus - beetle interactions and details of their life cycles.

\section{Materials and methods}

Polypores and beetles were collected in forest compartments (geoinformation data of the Finnish Forest and Park Service). These compartments have been outlined so as to include fairly uniform stands of forest, and their areas and shapes vary according to the terrain.

In the field a complete list of polypore species was made from each forest compartment visited. These basic data were used to estimate the prevalences (frequencies of occurrence) of the species. The commonest, easily identified species were recorded in situ, but rare and difficult species and members of critical genera were collected for a closer scrutiny. Collected specimens were preliminarily studied in the microscope before drying. Collections were dried in mushroom dryers with ventilated air at $+40-45^{\circ} \mathrm{C}$. When needed, the identifications were later reconfirmed in laboratory with a research microscope, sections mounted in Cotton Blue or Melzer's reagent, and studied at $\times 1250$ magnification and phase contrast illumination. In addition to polypores, also other wood-inhabiting fungi were observed and collected, in particular rare and threatened species.

Phellinus igniarius is treated here in a wide sense (including $P$. alni, $P$. cinereus, $P$. nigricans), and Postia lactea and $P$. tephroleuca are listed collectively under the name $P$. tephroleuca.

Beetle imagines and larvae were collected separately and preserved in $70 \%$ alcohol for further identification. Ecological parameters were recorded in the field: basidiocarp hardness, which is both a characteristic of each polypore species and a result of decomposition process; moisture conditions; the presence of anamorphic fungi; decomposition stages of the basidiocarps (Thunes 1994). We used four visually defined successive stages of decomposition (Table 1, Figs. 5-8), omitting stage $\mathrm{V}$ in sampling, i. e. when detached basidiocarps are already dwelled by soil organisms. We would propose the use of subdivisions D (dry) and W (wet) for classes II-IV, while they were adopted by Thunes (1994) for the class IV only. Insect impact seems to be a result of insect attraction rather than pre-colonizing condition, so we modified the definitions of decomposition stages (Table 1).

Fruit bodies with beetle larvae were taken in plastic bags and boxes together with substrate for rearing, kept for 2-3 months in $+4^{\circ} \mathrm{C}$, and then exposed in room temperature for further two months before the checking of the rearing results. Breeding records meet the criteria set by Lawrence (1973: 165). The commonest Finnish polypores (Niemelä 2003a) with comparatively well known coleopteran fauna were not surveyed. 
Table 1. Decomposition stages of polypore basidiocarps, according to Thunes (1994), modified. Subdivision D (dry) and W (wet) can be used for classes II-IV, if relevant.

\begin{tabular}{ll}
\hline Decomposition stage & Description \\
\hline I & Alive, fresh and still actively growing basidiocarp. \\
II & Alive, fully grown, mature basidiocarp. \\
III & $\begin{array}{l}\text { Dead, but fairly well-preserved basidiocarp; original outer and } \\
\text { inner structures are still easily seen. }\end{array}$ \\
IV & $\begin{array}{l}\text { Dead, strongly decomposed basidiocarp, structure transformed } \\
\text { into unorganised mass. }\end{array}$ \\
V & $\begin{array}{l}\text { Dead, detached and fallen basidiocarp, in transition of } \\
\text { becoming incorporated in soil. }\end{array}$ \\
\hline
\end{tabular}
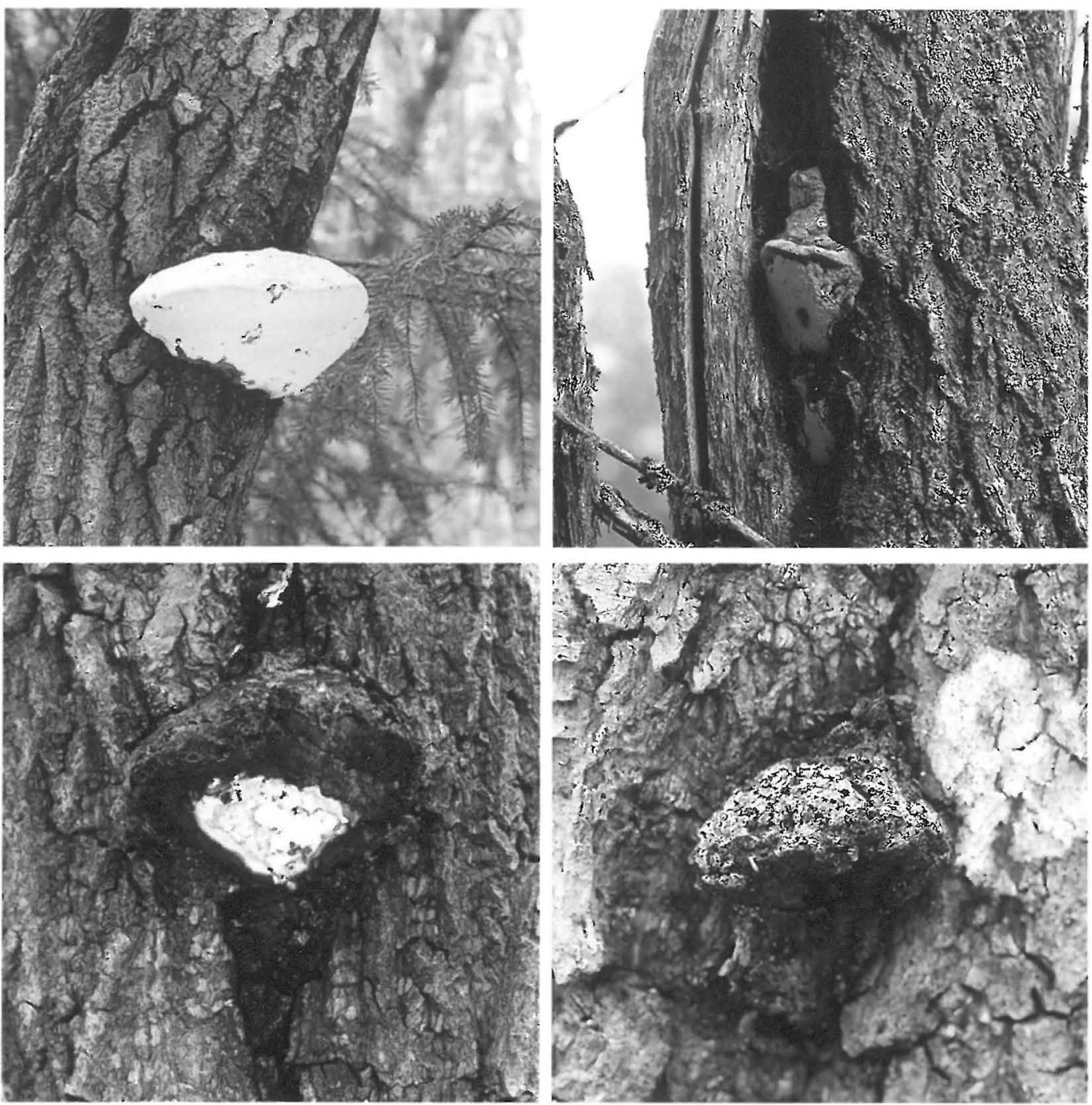

Figs. 5-8. Consecutive stages (I-IV) in the decomposition of the fruit body of Haploporus odorus. Photographs DSS, Pisavaara Strict Nature Reserve, northern Finland, 2003. 
Fungal collections are preserved in the Herbarium of the Botanical Museum, Finnish Museum of Natural History, University of Helsinki $(\mathrm{H})$; their nomenclature follows Niemelä (2004). Beetles will be donated after their investigation to the Zoological Museum, Finnish Museum of Natural History, University of Helsinki; single specimens have been given to identifiers (see Acknowledgements). Beetle nomenclature follows Silfverberg (1992), but the Ciidae are according to Müller et al. (2001). Authors of the Latin names of both fungi and beetles are given in Tables 2 and 3, respectively, and they are not repeated in the text.

All authors took part in the field work. This paper was prepared so that DSS wrote most of the text and all entomological sections. MS wrote parts of Introduction, and TN supervised the writing and wrote sections on fungal taxonomy.

\section{Results}

\subsection{Polypores}

Of the 105 polypore species collected by the authors, 29 are red-listed (Rassi et al. 2001) as belonging to different IUCN threat categories (2 EN - Piloporia sajanensis, Antrodia crassa; $11 \mathrm{VU}$; 16 NT; for details see Table 2). The list of species found by us is presented in Table 2, completed with some additional information. Our data to- gether with finds published before (Bondarceva et al. 1995; Bondartseva et al. 1998, 1999, 2001) make a total of 121 polypore species known by now from the region of study.

\subsection{Beetles}

In our study 115 polypore-associated beetle species were found (Table 3 ), including 24 species previously unrecorded for the Reserve, in addition to the 91 known ones (Yakovlev et al. 2001). 2614 beetle adults and larvae were collected. No red-listed beetles were found or reared from polypores. Beetles or their larvae were found in 52 (49.5\%) polypore species, while 53 polypore species appeared uninhabited (Table 2).

\section{Discussion}

\subsection{Polypores and other fungi}

The number of polypores found by us, 105 species (supplemented by 16 further species reported by others), is very high for any Finnish forest area. A recent inventory and herbarium study from the Pisavaara Strict Nature Reserve (Niemelä et

Table 2. Polypores (plus selected other Basidiomycota) and their beetles in the North Karelian Biosphere Reserve. Polypore species are given in an alphabetical order. IUCN threat categories according to Rassi et al. (2001) specified after polypore names if present. $\mathrm{N}=$ number of compartments where found; $\mathrm{A} / \mathrm{B}=$ number of fruit bodies inhabited/ studied. Numbers of insect specimens found or reared are given in parentheses after the beetle species name. Imagines collected in nature indicated light face; insect records of larvae or reared imagines in bold face. Beetle - fungus associations meeting the Lawrence (1973) criterion are marked with "10+" instead of the number of larvae or reared imago specimens.

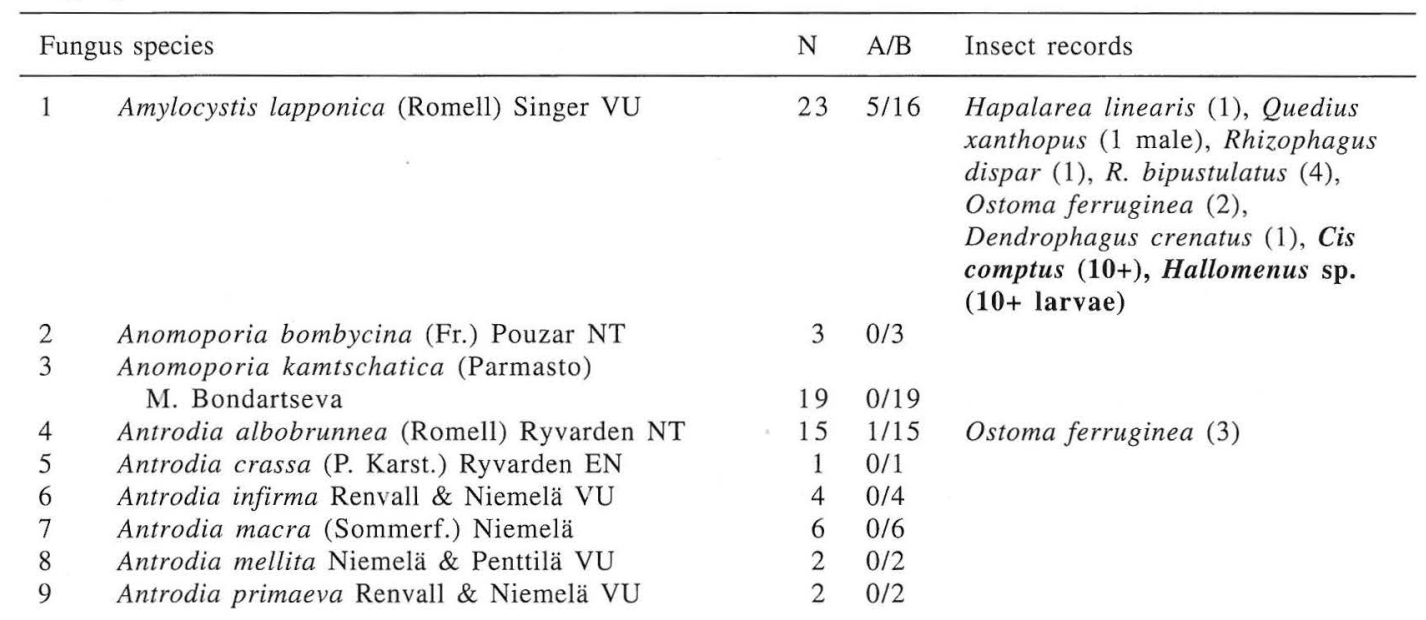




\begin{tabular}{|c|c|c|c|c|}
\hline \multicolumn{2}{|c|}{ Fungus species } & \multirow{2}{*}{$\frac{N}{7}$} & \multirow{2}{*}{$\frac{\mathrm{A} / \mathrm{B}}{2 / 7}$} & \multirow{2}{*}{$\begin{array}{l}\text { Insect records } \\
\text { Stenus carbonarius (1 female), Cis } \\
\text { hispidus (10+), Dolichocis } \\
\text { laricinus (2) }\end{array}$} \\
\hline 10 & Antrodia pulvinascens (Pilát) Niemelä VU & & & \\
\hline 11 & Antrodia serialis (Fr.) Donk & 43 & $1 / 8$ & Cis dentatus (3) \\
\hline 12 & Antrodia sinuosa (Fr.) P. Karst. & 42 & $0 / 42$ & \\
\hline 13 & Antrodia xantha (Fr. : Fr.) Ryvarden & 52 & $0 / 52$ & \\
\hline 14 & Antrodiella faginea Vampola \& Pouzar & 2 & $0 / 2$ & \\
\hline \multirow[t]{2}{*}{15} & Antrodiella semisupina (Berk. \& M.A. Curtis) & & & \\
\hline & Ryvarden & 10 & $3 / 10$ & $\begin{array}{l}\text { Acrulia inflata ( } 3 \text { males), } \\
\text { Rhizophagus dispar (1), } \\
\text { Cis boleti (1) }\end{array}$ \\
\hline 16 & Bjerkandera adusta (Willd. : Fr.) P. Karst. & 8 & $0 / 8$ & \\
\hline 17 & Byssoporia mollicula (Bourdot) Larsen \& Zak & 6 & $0 / 6$ & \\
\hline 18 & Ceriporia viridans (Berk. \& Broome) Donk & 2 & $0 / 2$ & \\
\hline 19 & Ceriporiopsis resinascens (Romell) Domański & 7 & $1 / 7$ & $\begin{array}{l}\text { Cis hispidus }(10+), \text { Octotemnus } \\
\text { glabriculus (2) }\end{array}$ \\
\hline 20 & Cerrena unicolor (Bull. : Fr.) Murrill & 17 & $1 / 17$ & Dromius sigma (1) \\
\hline 21 & Coltricia perennis (L. : Fr.) Murrill & 2 & $0 / 2$ & \\
\hline 22 & Daedaleopsis septentrionalis (P. Karst.) Niemelä & 1 & $0 / 1$ & \\
\hline 23 & Dichomitus squalens (P. Karst.) D.A. Reid NT & 8 & $2 / 8$ & $\begin{array}{l}\text { Scaphisoma boreale }(1), \\
\text { Ennearthron cornutum }(\mathbf{1 0 +})\end{array}$ \\
\hline 24 & Diplomitoporus crustulinus (Bres.) Domański NT & 3 & $0 / 3$ & \\
\hline 25 & Diplomitoporus lindbladii (Berk.) Gilb. \& Ryvarden & 4 & $0 / 4$ & \\
\hline 26 & Fomes fomentarius (L. : Fr.) Fr. & 59 & $1 / 1$ & Bolitophagus reticulatus $(10+)$ \\
\hline 27 & Fomitopsis pinicola (Sw. : Fr.) P. Karst. & 57 & $3 / 57$ & $\begin{array}{l}\text { Ostoma ferruginea }(10+) \\
\text { Rhizophagus dispar }(2) \\
\text { Atomaria affinis }(2)\end{array}$ \\
\hline 28 & Fomitopsis rosea (Alb. \& Schwein. : Fr.) P. Karst. NT & 33 & $5 / 33$ & $\begin{array}{l}\text { Ostoma ferruginea (4), } \\
\text { Tineidae G. sp. (1) }\end{array}$ \\
\hline 29 & Ganoderma lipsiense (Batsch) G.F. Atk. & 6 & $0 / 6$ & \\
\hline 30 & Gelatoporia pannocincta (Romell) Niemelä NT & 9 & $4 / 9$ & $\begin{array}{l}\text { Rhizophagus dispar (2), } \\
\text { Acrulia inflata ( } 1 \text { male, } 1 \text { female), } \\
\text { Sepedophilus testaceus (1 female), } \\
\text { Agathidium sp. (1), } \\
\text { Tineidae G. sp. (5) }\end{array}$ \\
\hline 31 & Gloeophyllum odoratum (Wulfen : Fr.) Imazeki & 1 & $0 / 1$ & \\
\hline 32 & Gloeophyllum sepiarium (Wulfen : Fr.) P. Karst. & 30 & $1 / 3$ & Cis comptus (9), Sulcacis affinis (1) \\
\hline 33 & Gloeoporus dichrous (Fr. : Fr.) Bres. & 15 & $6 / 8$ & $\begin{array}{l}\text { Scaphisoma agaricinum (1) } \\
\text { Cis comptus (11) }\end{array}$ \\
\hline 34 & Gloeoporus taxicola (Pers. : Fr.) Gilb. \& Ryvarden & 4 & $0 / 4$ & \\
\hline 35 & Hapalopilus rutilans (Pers. : Fr.) P. Karst. & 5 & $0 / 5$ & \\
\hline 36 & Heterobasidion parviporum Niemelä \& Korhonen & 2 & $0 / 2$ & \\
\hline 37 & Hyphodontia latitans (Bourd. \& Galz.) E. Langer & 1 & $0 / 1$ & \\
\hline 38 & Inonotus obliquus (Pers. : Fr.) Pilát & 50 & $0 / 50$ & $\begin{array}{l}\text { Rhizophagus bipustulatus (1), } \\
R . \text { dispar (1), Dorcatoma dresdensis } \\
(\mathbf{1 0 +}), \text { Triplax russica }(\mathbf{1}) \\
\text { Elateridae G. sp. (1 dead larva), } \\
\text { Abdera affinis (2), Mycetophagus } \\
\text { quadripustulatus (1) }\end{array}$ \\
\hline 39 & Inonotus radiatus (Sowerby : Fr.) P. Karst. & 2 & $0 / 2$ & Abdera affinis (3) \\
\hline 40 & Inonotus rheades (Pers.) P. Karst. & 11 & $5 / 11$ & $\begin{array}{l}\text { Corticaria rubripes }(1), \\
\text { Epuraea variegata }(1), \\
\text { Cis lineatocribratus }(\mathbf{1 0 +}) \text {, } \\
\text { Dorcatoma dresdensis }(\mathbf{1})\end{array}$ \\
\hline 41 & Ischnoderma benzoinum (Wahlenb.: Fr.) P. Karst. & 11 & $0 / 11$ & \\
\hline 42 & Junghuhnia luteoalba (P. Karst.) Ryvarden & 13 & $1 / 13$ & Sepedophilus testaceus (1 male) \\
\hline 43 & Lenzites betulinus (L. : Fr.) Fr. & 1 & $1 / 1$ & $\begin{array}{l}\text { Dinaraea aequata (1 male), } \\
\text { Cis hispidus }(\mathbf{1 0 +})\end{array}$ \\
\hline 44 & Leptoporus mollis (Pers. : Fr.) Quél. & 5 & $0 / 5$ & \\
\hline
\end{tabular}




\begin{tabular}{|c|c|c|c|c|}
\hline \multicolumn{2}{|c|}{ Fungus species } & \multirow{2}{*}{$\frac{\mathrm{N}}{1}$} & \multirow{2}{*}{$\frac{\mathrm{A} / \mathrm{B}}{0 / 1}$} & \multirow[t]{2}{*}{ Insect records } \\
\hline 45 & Oligoporus rennyi (Berk. \& Broome) Donk & & & \\
\hline 46 & Oligoporus sericeomollis (Romell) M. Bondartseva & 25 & $0 / 25$ & \\
\hline 47 & Parmastomyces mollissimus (Maire) Pouzar VU & 1 & $0 / 1$ & \\
\hline 48 & Perenniporia subacida (Peck) Donk NT & 4 & $0 / 4$ & \\
\hline 49 & Phaeolus schweinitzii (Fr.) Pat. & 2 & $2 / 2$ & $\begin{array}{l}\text { Atheta boleticola ( } 1 \text { male, } 1 \text { female) } \\
\text { Dorcatoma sp. }(6)\end{array}$ \\
\hline 50 & Phellinus chrysoloma (Fr.) Donk & 29 & $5 / 7$ & $\begin{array}{l}\text { Abdera flexuosa (1), Ennearthron } \\
\text { cornutum }(\mathbf{1 0 + )}\end{array}$ \\
\hline 51 & Phellinus conchatus (Pers. : Fr.) Quél. & 13 & $4 / 13$ & $\begin{array}{l}\text { Rhizophagus dispar (1), Sulcacis } \\
\text { affinis (2), Cis hispidus (2), } \\
\text { Dorcatoma sp. (10+), } \\
\text { Tineidae G. sp. (1) }\end{array}$ \\
\hline 52 & Phellinus ferrugineofuscus (P. Karst.) Bourdot NT & 27 & $1 / 4$ & Ciidae G. sp. (4 larvae) \\
\hline 53 & Phellinus igniarius (L. : Fr.) Quél. & 51 & $3 / 4$ & $\begin{array}{l}\text { Ennearthron cornutum }(10+) \\
\text { Dorcatoma dresdensis }(10+)\end{array}$ \\
\hline 54 & Phellinus laevigatus (P. Karst.) Bourdot \& Galzin & 27 & $2 / 4$ & $\begin{array}{l}\text { Rhizophagus dispar (4), } \\
\text { Acrulia inflata (1 male), } \\
\text { Orthoperus atomus (1), } \\
\text { Dorcatoma sp. (3) }\end{array}$ \\
\hline 55 & Phellinus lundellii Niemelä & 21 & $2 / 21$ & $\begin{array}{l}\text { Ennearthron cornutum (1), } \\
\text { Dorcatoma dresdensis (3), } \\
\text { Tineidae G. sp. (1) }\end{array}$ \\
\hline 56 & Phellinus nigrolimitatus (Romell) Bourdot \& Galzin & 17 & $0 / 17$ & \\
\hline 57 & Phellinus pini (Brot. : Fr.) A. Ames & 40 & $6 / 14$ & $\begin{array}{l}\text { Phloeocharis subtilissima } \\
(3 \text { females }+2) \text {, } \\
\text { Hallomenus binotatus }(1), \\
\text { Ennearthron cornutum }(\mathbf{1 0 +})\end{array}$ \\
\hline 58 & Phellinus populicola Niemelä & 10 & $0 / 10$ & Dorcatoma dresdensis $(10+)$ \\
\hline 59 & Phellinus punctatus (P. Karst.) Pilát & 2 & $0 / 2$ & Rhizophagus dispar (1) \\
\hline 60 & $\begin{array}{l}\text { Phellinus tremulae (Bondartsev) } \\
\text { Bondartsev \& Borisov }\end{array}$ & 44 & $0 / 5$ & \\
\hline 61 & Phellinus viticola (Schwein. ex Fr.) Donk & 45 & $1 / 7$ & $\begin{array}{l}\text { Cis boleti (1), Octotemnus } \\
\text { glabriculus ( } 3) \text {, } \\
\text { Ennearthron cornutum }(\mathbf{1 0 + )}\end{array}$ \\
\hline 62 & Physisporinus vitreus (Pers. : Fr.) P. Karst. & 2 & $0 / 2$ & \\
\hline 63 & Piloporia sajanensis (Parmasto) Niemelä EN & 1 & $0 / 1$ & \\
\hline 64 & Piptoporus betulinus (Bull. : Fr.) P. Karst. & 47 & $1 / 1$ & $\begin{array}{l}\text { Glischrochilus hortensis (1), } \\
\text { Cis bidentatus }(\mathbf{1 0 +}) \\
\text { Diaperis boleti }(\mathbf{1 0 +})\end{array}$ \\
\hline 65 & Polyporus brumalis (Pers. : Fr.) Fr. & 8 & $2 / 8$ & $\begin{array}{l}\text { Scaphisoma agaricinum (1), } \\
\text { Tineidae G. } \mathbf{s p .} \text { (1) }\end{array}$ \\
\hline 66 & Polyporus ciliatus Fr. : Fr. & 3 & $0 / 3$ & Rhizophagus dispar (1) \\
\hline 67 & Polyporus leptocephalus (Jacq. : Fr.) Fr. & 10 & $3 / 10$ & $\begin{array}{l}\text { Orthoperus corticalis (1), } \\
\text { Dolichocis laricinus (1), } \\
\text { Cis jacquemartii (2), } \\
\text { Cis lineatocribratus (1) }\end{array}$ \\
\hline 68 & Postia alni Niemelä \& Vampola & 15 & $2 / 15$ & $\begin{array}{l}\text { Hallomenus sp. (2 larvae), } \\
\text { Tineidae G. sp. (2) }\end{array}$ \\
\hline 69 & Postia caesia (Schrad. : Fr.) P. Karst. & 11 & $0 / 11$ & \\
\hline 70 & Postia fragilis (Fr.) Jülich & 5 & $2 / 5$ & $\begin{array}{l}\text { Hapalarea linearis (1), } \\
\text { Hallomenus sp. (2 larvae) }\end{array}$ \\
\hline 71 & Postia guttulata (Peck) Jülich NT & 7 & $0 / 7$ & \\
\hline 72 & Postia hibernica (Berk. \& Broome) Jülich NT & 1 & $0 / 1$ & \\
\hline 73 & Postia lateritia Renvall VU & 6 & $3 / 6$ & $\begin{array}{l}\text { Hapalarea linearis (1), } \\
\text { Lordithon lunulatus ( } 1 \text { male), } \\
\text { Hallomenus ?binotatus (2 larvae) }\end{array}$ \\
\hline 74 & Postia leucomallella (Murrill) Jülich & 19 & $3 / 19$ & $\begin{array}{l}\text { Hapalarea linearis (1 male), } \\
\text { Rhizophagus dispar (1), } \\
\text { Hallomenus sp. ( } 7 \text { larvae) }\end{array}$ \\
\hline
\end{tabular}




\begin{tabular}{|c|c|c|c|c|}
\hline \multicolumn{2}{|c|}{ Fungus species } & \multirow{2}{*}{$\mathrm{N}$} & \multirow{2}{*}{$\frac{\mathrm{A} / \mathrm{B}}{1 / 10}$} & \multirow{2}{*}{$\begin{array}{l}\text { Insect records } \\
\text { Ischnoglossa prolixa (1), } \\
\text { Rhizophagus dispar (1), } \\
\text { Hallomenus sp. (2 larvae) }\end{array}$} \\
\hline 75 & Postia placenta (Fr.) M.J. Larsen \& Lombard NT & & & \\
\hline 76 & Postia septentrionalis (Vampola) Renvall NT & 1 & $0 / 1$ & \\
\hline 77 & Postia stiptica (Pers. : Fr.) Jülich & 2 & $1 / 2$ & Hallomenus sp. (2 larvae) \\
\hline 78 & Postia tephroleuca (Fr.) Jülich & 16 & $2 / 16$ & $\begin{array}{l}\text { Acrulia inflata ( } 1 \text { female), } \\
\text { Atrecus pilicornis (1), } \\
\text { Rhizophagus dispar (3), } \\
\text { Hallomenus binotatus (2), } \\
\text { H. sp. (7 larvae) }\end{array}$ \\
\hline 79 & Postia undosa (Peck) Jülich & 4 & $0 / 4$ & \\
\hline 80 & Protomerulius caryae (Schwein.) Ryvarden VU & 5 & $0 / 5$ & \\
\hline 81 & Pycnoporellus fulgens (Fr.) Donk & 2 & $0 / 2$ & \\
\hline 82 & Pycnoporus cinnabarinus (Jacq. : Fr.) P. Karst. & 9 & $4 / 9$ & Sulcacis affinis (11) \\
\hline 83 & Rigidoporus corticola (Fr.) Pouzar & 28 & $9 / 12$ & $\begin{array}{l}\text { Agathidum pisanum (2), } \\
\text { Rhizophagus dispar (1), } \\
\text { Acrulia inflata ( } 3 \text { males, } 1 \text { female), } \\
\text { Staphylinidae G. sp. (10+ larvae), } \\
\text { Elateridae G. sp. (1) }\end{array}$ \\
\hline \multirow[t]{2}{*}{84} & Sarcoporia salmonicolor (Berk. \& & & & \\
\hline & M.A. Curtis) Teixeira NT & 2 & $1 / 2$ & Cis dentatus (6) \\
\hline 85 & Skeletocutis amorpha (Fr.) Kotl. \& Pouzar & 13 & $0 / 13$ & \\
\hline 86 & Skeletocutis biguttulata (Romell) Niemelä & 14 & $0 / 14$ & \\
\hline 87 & Skeletocutis brevispora Niemelä VU & 6 & $0 / 6$ & \\
\hline 88 & Skeletocutis carneogrisea A. David & 4 & $0 / 4$ & \\
\hline 89 & Skeletocutis kuehneri A. David & 4 & $0 / 4$ & \\
\hline 90 & Skeletocutis lenis (P. Karst.) Niemelä VU & 2 & $0 / 2$ & \\
\hline 91 & Skeletocutis odora (Sacc.) Ginns NT & 6 & $1 / 6$ & Staphylinidae G. sp. (10+ larvae) \\
\hline 92 & Skeletocutis papyracea A. David & 5 & $0 / 5$ & \\
\hline 93 & Skeletocutis stellae (Pilát) Jean Keller VU & 4 & $0 / 4$ & \\
\hline 94 & Trametes hirsuta (Wulfen : Fr.) Pilát & 1 & $1 / 1$ & $\begin{array}{l}\text { Cis hispidus }(10+) \text {, } \\
\text { Octotemnus glabriculus }(10+)\end{array}$ \\
\hline 95 & Trametes ochracea (Pers.) Gilb. \& Ryvarden & 38 & $5 / 5$ & $\begin{array}{l}\text { Cis boleti }(10+), \text { Cis hispidus }(10+), \\
\text { Octotemnus glabriculus }(10+), \\
\text { Tineidae G. sp. (1) }\end{array}$ \\
\hline 96 & Trametes pubescens (Schumach. : Fr.) Pilát & 10 & $4 / 4$ & Cis boleti $(10+)$, Cis hispidus $(10+)$ \\
\hline 97 & Trametes velutina (Fr.) G. Cunn. & 4 & $3 / 4$ & $\begin{array}{l}\text { Cis boleti }(10+) \text {, Octotemnus } \\
\text { glabriculus }(10+) \text {, } \\
\text { Tineidae G. sp. (1) }\end{array}$ \\
\hline 98 & Trechispora candidissima (Schwein.) Bondartsev & 1 & $0 / 1$ & \\
\hline \multirow[t]{2}{*}{99} & Trechispora hymenocystis (Berk. \& Broome) & & & \\
\hline & K.-H. Larsson & 1 & $0 / 1$ & \\
\hline 100 & Trechispora mollusca (Pers. : Fr.) Liberta & 4 & $0 / 4$ & \\
\hline 101 & Trichaptum abietinum (Pers. : Fr.) Ryvarden & 53 & $0 / 53$ & \\
\hline \multirow[t]{2}{*}{102} & Trichaptum fuscoviolaceum (Ehrenb. : Fr.) & & & \\
\hline & Ryvarden & 19 & $1 / 2$ & Cis punctulatus $(10+)$ \\
\hline 103 & Trichaptum laricinum (P. Karst.) Ryvarden NT & 5 & $0 / 5$ & \\
\hline 104 & Trichaptum pargamenum (Fr.) G. Cunn. NT & 20 & $6 / 10$ & $\begin{array}{l}\text { Leptusa pulchella (1), } \\
\text { Acrulia inflata (1 male), } \\
\text { Rhizophagus dispar (1), } \\
\text { Cis comptus (8), } \\
\text { Cis lineatocribratus (1), } \\
\text { Ennearthron cornutum (2), } \\
\text { Cis jacquemartii (2), } \\
\text { Cis punctulatus (3) }\end{array}$ \\
\hline 105 & Tyromyces chioneus (Fr.) P. Karst. & 6 & $0 / 6$ & Atheta sp. (1) \\
\hline
\end{tabular}




\begin{tabular}{|c|c|c|c|c|}
\hline \multicolumn{2}{|c|}{ Fungus species } & $\mathrm{N}$ & $\mathrm{A} / \mathrm{B}$ & Insect records \\
\hline \multicolumn{5}{|c|}{ Non-polypore fungi } \\
\hline 106 & Amylostereum chailletii (Pers. : Fr.) Boidin & 1 & $0 / 1$ & \\
\hline 107 & Asterodon ferruginosus Pat. & 13 & $0 / 13$ & \\
\hline 108 & Basidioradulum radula (Fr.) Nobles & 5 & $0 / 5$ & \\
\hline 109 & Calocera cornea (Batsch. : Fr.) Fr. & 1 & & $0 / 1$ \\
\hline 110 & Cantharellus tubaeformis (Bull. : Fr.) Fr. & 1 & $0 / 1$ & \\
\hline 111 & Chaetoderma luna (Romell) Parmasto & 8 & $0 / 1$ & \\
\hline 112 & Chondrostereum purpureum (Pers. : Fr.) Pouzar & 8 & $0 / 1$ & \\
\hline 113 & Columnocystis abietina (Pers. : Fr.) Pouzar & 14 & $0 / 14$ & \\
\hline 114 & Coniophora olivacea (Pers. : Fr.) P. Karst. & 13 & $0 / 13$ & \\
\hline 115 & Creolophus cirrhatus (Pers. : Fr.) P. Karst. & 2 & $0 / 2$ & \\
\hline 116 & Cytidia salicina $(\mathrm{Fr}$.$) Burt$ & 1 & $0 / 1$ & \\
\hline 117 & Daldinia concentrica (Bolton : Fr.) Ces. \& De Not s.l. & 1 & $0 / 1$ & \\
\hline 118 & Gloiodon strigosus (Schwein. : Fr.) P. Karst. VU & 3 & $0 / 3$ & \\
\hline 119 & Hericium coralloides (Scop. : Fr.) Pers. & 9 & $0 / 9$ & \\
\hline 120 & Hydnellum aurantiacum (Batsch : Fr.) P. Karst. & 1 & $0 / 1$ & \\
\hline 121 & Hydnellum ferrugineum (Fr. : Fr.) P. Karst. & 10 & $0 / 10$ & \\
\hline 122 & Hydnellum gracilipes (P. Karst.) P. Karst. & 1 & $0 / 1$ & \\
\hline 123 & Hypochnicium multiforme (Berk. \& Broome) Hjortst. & 1 & $0 / 1$ & \\
\hline 124 & Hypsizygus ulmarius (Bull.) Redhead & 1 & $1 / 1$ & Rhizophagus dispar (10+) \\
\hline 125 & Kavinia alboviridis (Morgan) Gilb. \& Budington NT & 1 & $0 / 1$ & \\
\hline 126 & Laeticorticium roseum (Fr.) Donk & 10 & $0 / 10$ & \\
\hline 127 & Laxitextum bicolor (Pers. : Fr.) Lentz & 3 & $0 / 3$ & \\
\hline 128 & Lentaria epichnoa (Fr.) Corner & 2 & $0 / 2$ & \\
\hline 129 & Lentinellus vulpinus (Sowerby) Kühner \& Maire & 3 & $0 / 3$ & \\
\hline 130 & Mycena tintinabulum Quél. VU & 1 & $0 / 1$ & \\
\hline 131 & Mycoacia fuscoatra (Fr. : Fr.) Donk & 7 & $0 / 7$ & \\
\hline 132 & Panellus serotinus (Schrad. : Fr.) Kühner & 1 & $0 / 1$ & \\
\hline 133 & Phanerochaete sanguinea (Fr.) Pouzar & 2 & $0 / 2$ & \\
\hline 134 & Phellodon niger (Fr. : Fr.) P. Karst. & 3 & $0 / 3$ & \\
\hline 135 & Phellodon tomentosus (L. : Fr.) Banker & 1 & $0 / 1$ & \\
\hline 136 & Phellodon secretus Niemelä \& Kinnunen & 2 & $0 / 2$ & \\
\hline 137 & Phlebia centrifuga P. Karst. VU & 9 & $0 / 9$ & \\
\hline 138 & Phlebia cornea (Bourd. \& Galzin) Parmasto NT & 2 & $0 / 2$ & \\
\hline 139 & Phlebia radiata $\mathrm{Fr}$. & 3 & $0 / 3$ & \\
\hline 140 & Phlebia tremellosa (Schrad. : Fr.) Burds. \& Nakasone & 18 & $0 / 18$ & \\
\hline 141 & Pholiota heteroclita (Fr. : Fr.) Quél. & 1 & $0 / 1$ & \\
\hline 142 & Phyllotopsis nidulans (Pers. : Fr.) Singer & 3 & $0 / 3$ & \\
\hline 143 & Pleurotus dryinus (Pers. : Fr.) P. Kumm. & 1 & $1 / 1$ & $\begin{array}{l}\text { Rhizophagus dispar }(10+) \\
\text { Sepedophilus testaceus }(3), \\
\text { Hapalaraea melanocephala (1) }\end{array}$ \\
\hline 144 & Pleurotus pulmonarius (Fr.) Quél. & 6 & $6 / 6$ & $\begin{array}{l}\text { Rhizophagus dispar }(10+), \\
\text { R. bipustulatus }(10+), \\
\text { Mycetophagus multipunctatus (8), } \\
\text { Triplax aenea }(10+), \\
\text { Atheta picipes }(10+), \\
\text { Phloeopora testacea (3), } \\
\text { Cerylon sp. ( } 7)\end{array}$ \\
\hline 145 & Plicatura nivea (Sommerf. : Fr.) P. Karst. & 9 & $0 / 9$ & \\
\hline 146 & Pseudohydnum gelatinosum (Scop. : Fr.) P. Karst. & 2 & & \\
\hline 147 & Pseudomerulius aureus (Fr.) Jülich & 6 & $0 / 6$ & \\
\hline 148 & Punctularia strigosozonata (Schw.) Talbot & 1 & $0 / 1$ & \\
\hline 149 & Sarcodon squamosus (Schaeff.) Quél. & 3 & $0 / 3$ & \\
\hline 150 & Serpula himantioides (Fr. : Fr.) P. Karst. & 19 & $0 / 19$ & \\
\hline 151 & Sistotrema raduloides (P. Karst.) Donk & 10 & $0 / 10$ & \\
\hline 152 & Steccherinum ochraceum (Pers.) Gray & 1 & $0 / 1$ & \\
\hline 153 & Stereopsis vitellina (Plowr.) D.A. Reid NT & 1 & $0 / 1$ & \\
\hline
\end{tabular}




\begin{tabular}{lrll}
\hline Fungus species & N & A/B & Insect records \\
\hline 154 & Stereum hirsutum (Willd. : Fr.) Gray & 11 & $0 / 11$ \\
155 & Stereum rugosum Pers. : Fr. & 8 & $0 / 8$ \\
156 & Stereum sanguinolentum (Alb. \& Schwein. : Fr.) Fr. & 14 & $0 / 14$ \\
157 & Thelephora terrestris Ehrh. : Fr. & 3 & $0 / 3$ \\
\hline
\end{tabular}

Literature data, specimens not studied by us

\author{
Albatrellus confluens (Alb. \& Schwein. : Fr.) Kotl. \& Pouzar \\ Albatrellus ovinus (Schaeff. : Fr.) Kotl. \& Pouzar \\ Albatrellus subrubescens (Murrill) Pouzar \\ Antrodiella romellii (Donk) Niemelä \\ Bjerkandera fumosa (Pers. : Fr.) P. Karst. \\ Ceriporia reticulata (H. Hoffm. : Fr.) Domański \\ Datronia mollis (Sommerf.) Donk \\ Gloeophyllum protractum (Fr.) Imazeki \\ Haploporus odorus (Sommerf.) Bondartsev \& Singer \\ Hyphodontia flavipora (Cooke) Sheng H. Wu \\ Hyphodontia paradoxa (Schrad. : Fr.) E. Langer \& Vesterholt \\ Junghuhnia collabens (Fr.) Ryvarden \\ Junghuhnia lacera (P. Karst.) Niemelä \& Kinnunen \\ Polyporus badius (Pers.) Schwein. \\ Polyporus pseudobetulinus (Pilát) Thorn, Kotir. \& Niemelä \\ Rigidoporus populinus (Schumach, : Fr.) Pouzar
}

al. 2004) yielded 125 species; that reserve is often considered to be the richest site in the whole of West and Middle Fennoscandian coniferous forest zone.

We have inventoried several forest reserves during recent years from different parts of North and East Finland (Niemelä \& Dai 1998, Niemelä \& Dai 1999, Niemelä \& Kinnunen 2001, Niemelä \& Kinnunen 2002, Niemelä et al. 2002, Niemelä \& Kinnunen 2003). All they have been studied in the same way, by making full lists of polypore species from each forest compartment visited. The high number of compartments (593 compartments studied during the listed inventories, Table 4) enables us to make a summary on the commonest polypore species in these old and virgin forests. While most of the high-frequency species $(\mathrm{Fo}$ mes fomentarius, Trichaptum abietinum, Fomitopsis pinicola, etc.) are able to inhabit many kinds of wooded biotopes, strikingly many are inhabitants of old-growth forests, and have virtually disappeared from areas where forest management has been practiced, for instance, tree stands thinned, dead trees removed, etc. This is a good example on the impoverishing effect of modern forestry on forest biodiversity.

Our research site in the Koitajoki Reserve in eastern Finland lies at the transition between Middle Boreal and Southern Boreal zones (Ahti et al. 1968), in their slightly continental sections. The distributions of many northern species are known to reach further south in these climatically continental parts of East Fennoscandia, and similar results were obtained in our study, too. Among the polypores, Daedaleopsis septentrionalis and Trichaptum laricinum have typical northern distributions, and for instance in western Finland they are found almost exclusively within the Northern Boreal zone, i.e. in Lapland north of the Arctic Circle. However, they as well as some typically northerly hydnaceous fungi (Hydnellum gracilipes, Phellodon secretus; see notes below) and Corticiaceae (Phlebia centrifuga) were found in our research area, too.

Trichaptum pargamenum has a clearly eastern distribution in Europe, and it is lacking from Central and West Finland, as well as from Sweden, Denmark and Norway (Hansen \& Knudsen 
Table 3. Systematic list of beetles attracted to polypores; North Karelian Biosphere Reserve. Numbers refer to host fungi (Table 2); light face = records of beetle imagines; bold face $=$ larvae or rearings.

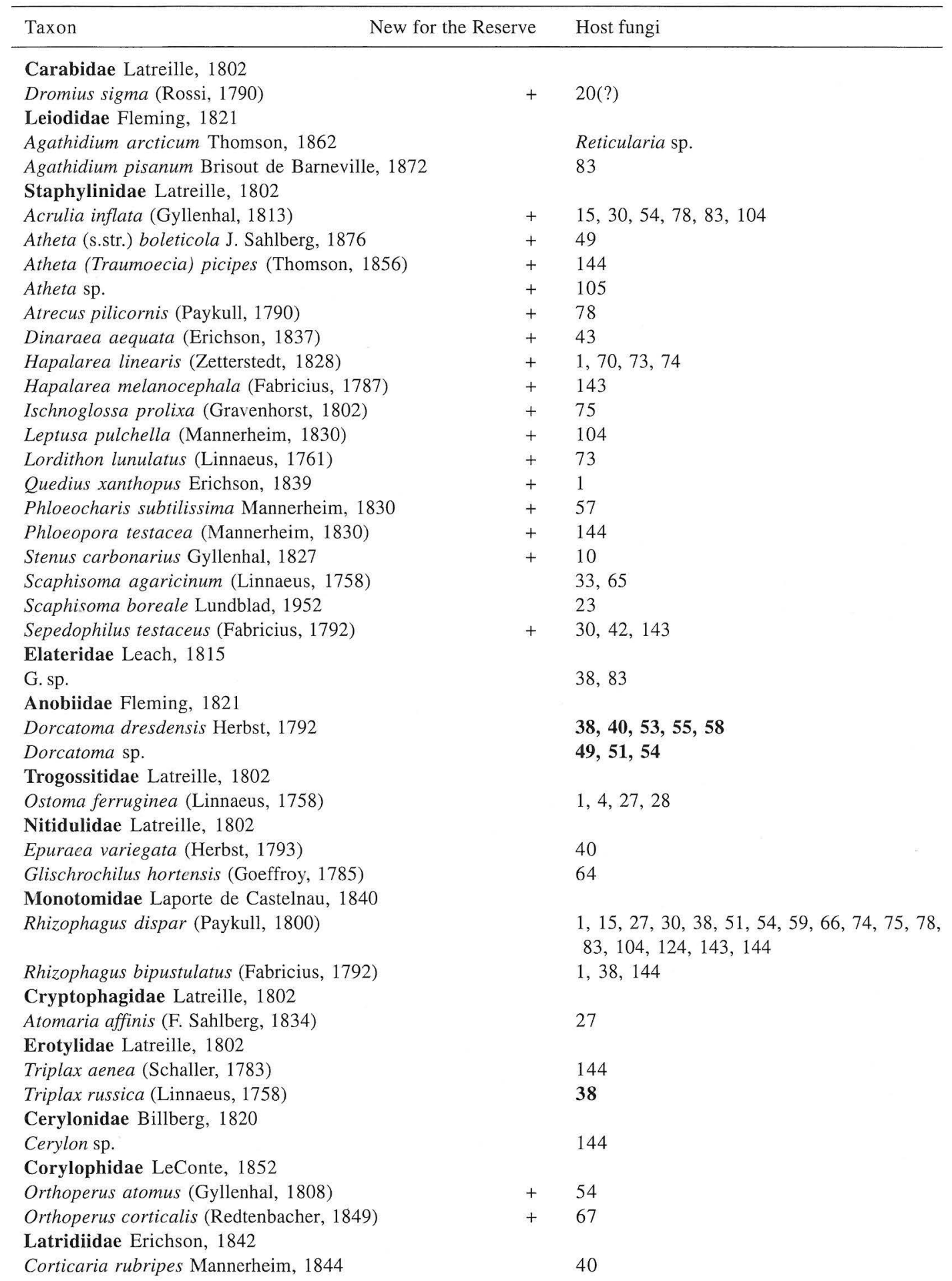




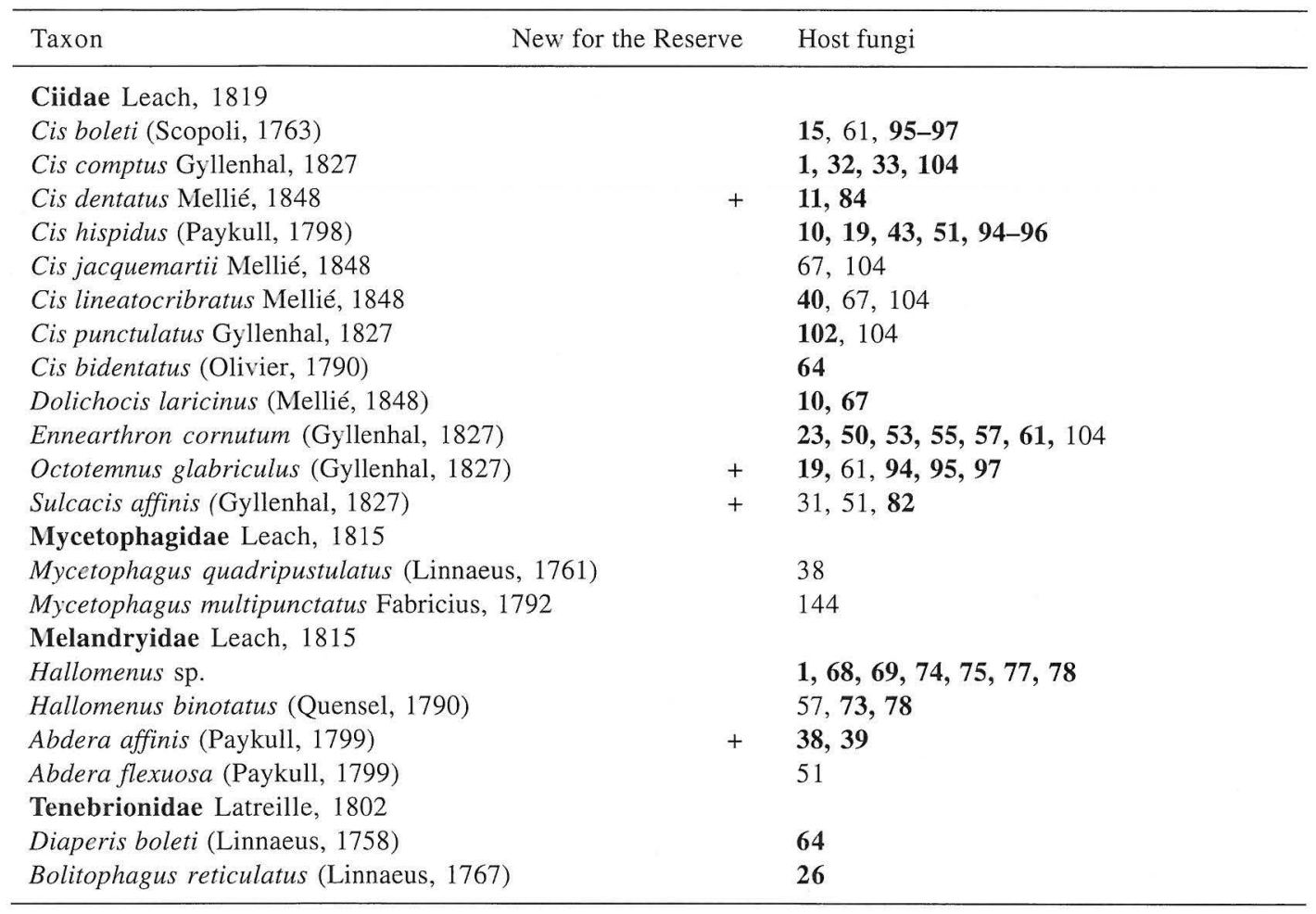

1997). In our research area it was found frequently on birch. Piloporia sajanensis seems to have a fairly continental, eastern distribution, too.

Some species of predominantly southern distribution were also found. Pycnoporellus fulgens is confined mostly to southern Fennoscandia (Niemelä 1980), but we recorded it once. This species has also been collected in Russian Karelia, not far from the Koitajoki area (Shubin \& Krutov 1979), and hence its finding was not unexpected. Among the non-poroid Aphyllophorales with a southern distribution, we found Punctularia strigosozonata, Steccherinum ochraceum, Stereopsis vitellina, and among Agaricales Mycena tintinabulum, living on fallen trunk of birch.

Our records include a great number of threatened species: 2 endangered (EN), 11 vulnerable (VU), and 16 near-threatened (NT). They are indicated in the species list (Table 2). We made notes on the other wood-inhabiting fungi only in passing, when time allowed. Some threatened species were found: Mycena tintinabulum (VU), Gloiodon strigosus (VU), Kavinia alboviridis (NT), Phlebia centrifuga (VU), Phlebia cornea (NT), Punctularia strigosozonata (CR), Sistotrema raduloides (NT) and Stereopsis vitellina (NT). These results clearly illustrate the high conservation values of the Koitajoki Natura 2000 site.

\subsection{Notes on selected fungi}

Antrodia crassa (Fig. 9) is extremely rare in Finland (Kotiranta \& Niemelä 1996), and almost totally confined to the oldest pine forests. However, every now and then the species is found in more mesic, spruce dominated forests, indicating that dry environment per se is not obligatory for the species to grow. In our Koitajoki inventory the species was found in the oldest and best-preserved forest patch, the Kelokkoaho forest, which arises like an island in the middle of vast peatlands. Host tree was an exceptionally thick (over $50 \mathrm{~cm}$ ), long- 
Table 4. The commonest polypore species in some protected old-growth forests of North and East Finland. The numbers were compiled from 8 inventories carried out in 1998-2004; their references are given in the text (Repovesi: unpubl.). TOTAL = The total of the inventories in 1998-2004. Luosto = Luosto fells in central Finnish Lapland; Ylläs = Yllästunturi and Aakenustunturi fells and highland in western Finnish Lapland; Koro = Korouoma Forest Reserve in northeastern Finland (Posio commune); Koita = Koitajoki Natura 2000 site; Pisa = Pisavaara Strict Nature Reserve (Rovaniemi commune); Repo = Repovesi National Park, Central Finland (Valkeala commune). $\mathrm{n}=$ The number of forestry compartments studied. \# = The order of frequency (prevalence) of the species in each inventory. $\%=$ In how many of the studied compartments (per cent) the species was found. Name in bold face $=$ An indicator of old or virgin forest (see Kotiranta \& Niemelä 1996).

\begin{tabular}{|c|c|c|c|c|c|c|c|c|c|c|c|c|c|c|c|c|c|c|}
\hline & \multicolumn{2}{|c|}{$\begin{array}{c}\text { TOTAL } \\
1998-2004 \\
n=593\end{array}$} & \multicolumn{2}{|c|}{$\begin{array}{c}\text { Luosto } \\
1998 \\
\mathrm{n}=79\end{array}$} & \multicolumn{2}{|c|}{$\begin{array}{l}\text { Ylläs } \\
1999 \\
n=72\end{array}$} & \multicolumn{2}{|c|}{$\begin{array}{l}\text { Ylläs } \\
2000 \\
\mathrm{n}=96\end{array}$} & \multicolumn{2}{|c|}{$\begin{array}{l}\text { Ylläs } \\
2001 \\
n=38\end{array}$} & \multicolumn{2}{|c|}{$\begin{array}{l}\text { Koro } \\
2001 \\
\mathrm{n}=65\end{array}$} & \multicolumn{2}{|c|}{$\begin{array}{l}\text { Koita } \\
2002 \\
n=70\end{array}$} & \multicolumn{2}{|c|}{$\begin{array}{c}\text { Pisa } \\
2003 \\
n=124\end{array}$} & \multicolumn{2}{|c|}{$\begin{array}{l}\text { Repo } \\
2004 \\
n=49\end{array}$} \\
\hline & $\#$ & $\%$ & $\#$ & $\%$ & \# & $\%$ & \# & $\%$ & $\#$ & $\%$ & $\#$ & $\%$ & $\#$ & $\%$ & $\#$ & $\%$ & \# & $\%$ \\
\hline Fomes fomentarius & 1 & 73.8 & 4 & 65.8 & 2 & 69.4 & 1 & 72.9 & 2 & 81.6 & 1 & 78.5 & 1 & 84.3 & 2 & 76.6 & 1 & 61.2 \\
\hline Trichaptum abietinum & 2 & 67.0 & 7 & 57.0 & 3 & 65.3 & 4 & 63.5 & 1 & 86.8 & 5 & 64.6 & 3 & 75.7 & 3 & 70.2 & 3 & 53.1 \\
\hline Fomitopsis pinicola & 3 & 66.0 & 5 & 60.8 & 4 & 58.3 & 5 & 56.3 & 6 & 71.1 & 6 & 63.1 & 2 & 81.4 & 1 & 81.5 & 2 & 55.1 \\
\hline Phellinus igniarius & 4 & 63.8 & 1 & 70.9 & 1 & 72.2 & 2 & 71.9 & 12 & 65.8 & 2 & 70.8 & 5 & 72.9 & 7 & 49.2 & 7 & 36.7 \\
\hline Inonotus obliquus & 5 & 60.6 & 3 & 68.4 & 6 & 55.6 & 3 & 67.7 & 9 & 68.4 & 7 & 58.5 & 6 & 71.4 & 5 & 55.7 & 5 & 38.8 \\
\hline Phellinus viticola & 6 & 57.3 & 6 & 59.5 & 7 & 54.2 & 6 & 55.2 & 4 & 76.3 & 4 & 64.6 & 8 & 64.3 & 4 & 66.1 & 18 & 18.4 \\
\hline Antrodia serialis & 7 & 50.2 & 16 & 34.2 & 8 & 54.2 & 12 & 40.6 & 13 & 63.2 & 3 & 64.6 & 10 & 61.4 & 10 & 46.8 & 6 & 36.7 \\
\hline Gloeophyllum sepiarium & 8 & 49.5 & 2 & 69.6 & 9 & 51.4 & 11 & 44.8 & 3 & 79.0 & 8 & 55.4 & 15 & 42.9 & 12 & 40.3 & 27 & 12.2 \\
\hline Piptoporus betulinus & 9 & 48.4 & 13 & 40.5 & 15 & 37.5 & 7 & 55.2 & 10 & 68.4 & 12 & 43.1 & 7 & 67.1 & 22 & 28.2 & 4 & 46.9 \\
\hline Fomitopsis rosea NT & 10 & 44.3 & 8 & 46.8 & 5 & 58.3 & 8 & 52.1 & 8 & 68.4 & 20 & 29.2 & 14 & 47.1 & 11 & 40.3 & 25 & 12.2 \\
\hline Antrodia xantha. & 11 & 44.2 & 11 & 41.8 & 16 & 33.3 & 15 & 38.5 & 22 & 36.8 & 9 & 52.3 & 4 & 74.3 & 8 & 47.6 & 13 & 28.6 \\
\hline Trametes ochracea & 12 & 42.0 & 21 & 26.6 & 14 & 44.4 & 16 & 36.5 & 7 & 71.1 & 18 & 32.3 & 13 & 54.3 & 15 & 36.3 & 9 & 34.7 \\
\hline Phellinus chrysoloma & 13 & 42.0 & 9 & 46.8 & 10 & 50.0 & 9 & 51.0 & 15 & 60.5 & 10 & 49.2 & 16 & 41.4 & 14 & 37.1 & - & 0 \\
\hline Phellinus nigrolimitatus & 14 & 37.3 & 15 & 36.7 & 13 & 43.1 & 13 & 40.6 & 5 & 73.7 & 11 & 44.6 & 28 & 24.3 & 17 & 33.1 & 71 & 2.0 \\
\hline Cerrena unicolor & 15 & 36.5 & 10 & 45.6 & 11 & 47.2 & 10 & 44.8 & 11 & 65.8 & 23 & 26.2 & 27 & 24.3 & 19 & 29.8 & 36 & 8.2 \\
\hline Amylocystis lapponica $\mathrm{VU}$ & 16 & 36.3 & 12 & 40.5 & 12 & 44.4 & 14 & 38.5 & 14 & 60.5 & 17 & 33.9 & 21 & 32.9 & 13 & 37.9 & 60 & 2.0 \\
\hline Antrodia sinuosa & 17 & 35.4 & 17 & 31.7 & 20 & 23.6 & 25 & 19.8 & 27 & 26.3 & 16 & 38.5 & 11 & 60.0 & 6 & 52.4 & 11 & 30.6 \\
\hline Phellinus tremulae & 18 & 34.3 & 24 & 21.5 & 23 & 22.2 & 24 & 20.1 & 32 & 23.7 & 14 & 41.5 & 9 & 62.9 & 9 & 47.6 & 8 & 34.7 \\
\hline Oligoporus sericeomollis & 19 & 31.4 & 20 & 26.6 & 27 & 18.1 & 18 & 31.2 & 19 & 42.1 & 15 & 40.0 & 20 & 35.7 & 16 & 34.7 & 15 & 22.5 \\
\hline Phel. ferrugineofuscus NT & 20 & 29.9 & 23 & 22.8 & 17 & 33.3 & 17 & 32.3 & 17 & 47.4 & 26 & 21.5 & 18 & 38.6 & 18 & 30.7 & 26 & 12.2 \\
\hline Trichaptum fuscoviolaceum & 21 & 26.9 & 18 & 31.7 & 28 & 18.1 & 19 & 29.2 & 26 & 29.0 & 21 & 29.2 & 26 & 27.1 & 23 & 28.2 & 16 & 22.5 \\
\hline Phellinus laevigatus & 22 & 25.2 & 29 & 13.9 & 19 & 29.2 & 26 & 19.8 & 24 & 36.8 & 24 & 24.6 & 19 & 38.6 & 21 & 28.2 & 29 & 10.2 \\
\hline Antrodia albobrunnea NT & 23 & 20.3 & 19 & 27.9 & 30 & 13.9 & 30 & 15.6 & 29 & 23.7 & 13 & 41.5 & 30 & 21.4 & 26 & 16.1 & 63 & 2.0 \\
\hline Phellinus conchatus & 24 & 20.0 & 25 & 19.0 & 22 & 23.6 & 27 & 18.8 & 25 & 31.6 & 30 & 13.8 & 35 & 18.6 & 20 & 28.2 & 46 & 6.1 \\
\hline Onnia leporina & 25 & 18.9 & 14 & 38.0 & 18 & 30.6 & 21 & 26.0 & 20 & 42.1 & 29 & 13.9 & - & 0 & 96 & 0.8 & - & 0 \\
\hline Phellinus lundellii & 26 & 18.1 & 26 & 19.0 & 40 & 8.3 & 29 & 17.7 & 18 & 44.7 & 33 & 12.3 & 22 & 30.0 & 31 & 12.9 & - & 0 \\
\hline Postia caesia & 27 & 17.5 & 72 & 1.3 & 45 & 6.9 & 69 & 2.1 & 16 & 57.9 & 27 & 20.0 & 39 & 15.7 & 44 & 7.3 & 12 & 28.6 \\
\hline Gloeoporus dichrous & 28 & 15.9 & 27 & 17.7 & 21 & 23.6 & 34 & 10.4 & 28 & 26.3 & 47 & 4.6 & 31 & 21.4 & 38 & 8.9 & 21 & 14.3 \\
\hline Skeletocutis odora NT & 29 & 13.6 & 38 & 7.6 & 31 & 13.9 & 22 & 26.0 & 21 & 39.5 & 44 & 6.2 & 57 & 8.6 & 45 & 7.3 & - & 0 \\
\hline Coltricia perennis & 30 & 13.5 & 35 & 7.6 & 37 & 8.3 & 20 & 27.1 & 36 & 18.4 & 22 & 27.7 & 83 & 2.9 & 35 & 9.7 & 43 & 6.1 \\
\hline Phellinus pini & 31 & 13.4 & 33 & 10.1 & 50 & 5.6 & 66 & 2.1 & 74 & 2.6 & 43 & 7.7 & 12 & 57.1 & 27 & 16.1 & 47 & 6.1 \\
\hline Skeletocutis lenis VU & 32 & 12.0 & 31 & 11.4 & 47 & 6.9 & 31 & 14.6 & 34 & 23.7 & 19 & 30.8 & 99 & 2.9 & 51 & 5.7 & - & 0 \\
\hline Gloeoporus taxicola & 33 & 11.9 & 28 & 13.9 & 26 & 18.1 & 38 & 8.3 & 23 & 36.8 & 41 & 7.7 & 67 & 5.7 & 54 & 4.8 & - & 0 \\
\hline Trametes pubescens & 34 & 10.8 & 42 & 5.1 & 29 & 16.7 & 23 & 22.9 & 41 & 10.5 & 45 & 6.2 & 44 & 14.3 & 56 & 4.8 & 48 & 6.1 \\
\hline Climacocystis borealis & 35 & 10.7 & 32 & 10.1 & 25 & 18.1 & 32 & 11.5 & 31 & 23.7 & 39 & 7.7 & - & 0 & 89 & 14.5 & - & 0 \\
\hline Rigidoporus corticola & 36 & 10.7 & 76 & 1.3 & 87 & 1.4 & 93 & 1.0 & - & 0 & 28 & 16.9 & 17 & 40.0 & 30 & 14.5 & 32 & 10.2 \\
\hline Junghuhnia luteoalba & 37 & 10.7 & 70 & 1.3 & 43 & 6.9 & 47 & 5.2 & 53 & 7.9 & 36 & 10.8 & 34 & 18.6 & 24 & 20.2 & 22 & 14.3 \\
\hline Next species & $<$ & $10.0 \%$ & & & & & & & & & & & & & & & & \\
\hline
\end{tabular}


ago fallen trunk of pine, on which also Skeletocutis stellae was growing. Long continuity of the forest, undisturbed conditions, and a very old, thick, fallen kelo tree (see Niemelä et al. 2002) seem to be needed for $A$. crassa to survive.

Piloporia sajanensis (Fig. 10) is a rarity throughout its range. It belongs to the so-called successor species (Niemelä et al. 1995; see below), which mostly inhabit trees that have first been decayed by other fungi. Such successors are fairly specific in terms of their preceding species; Piloporia sajanensis lives almost exclusively on trees decayed by Trichaptum larici- num or, more seldom, the other Trichaptum species. Our sole find of $P$. sajanensis was growing on a fallen trunk of spruce, effectively white-rotted by $T$. laricinum, whose basidiocarps emerged in hundreds along the whole trunk. There are about 10 records of this vulnerable species in Finland, all of them from northern or easternmost parts of the country.

Hyphodontia latitans was reported from the same area already by Bondartseva et al. (1998). We recollected the species in another site of the Koitajoki Reserve, from a thin ( $9 \mathrm{~cm}$ diam.), strongly decayed fragment of spruce trunk. These are
Fig. 9. Fruit body of Antrodia crassa. Posio, Korouoma Nature Reserve, 2001, Niemelä 7085.

Fig. 10. Fruit body of Piloporia sajanensis. North Karelian Biosphere Reserve, Tapionaho, 2002, Niemelä 7496.
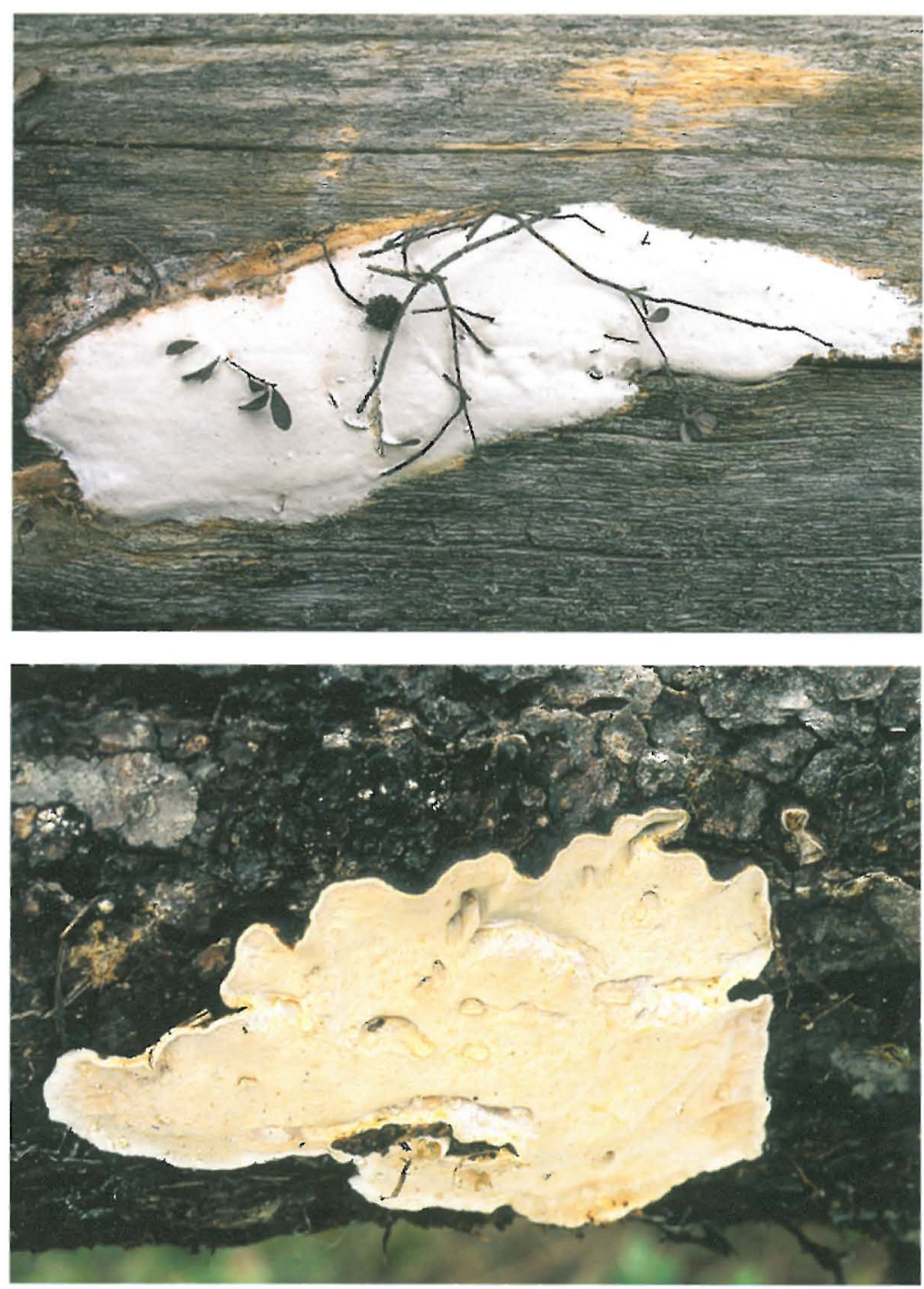
the only records of the species in Finland. The material was kindly identified by Heikki Kotiranta (Helsinki) and Karl-Henrik Larsson (Göteborg).

Hydnellum gracilipes is a rare, northern species of hydnaceous fungi. It was thoroughly described, illustrated and discussed by Kõljalg and Renvall (2000); at that time it was known from seven sites in Finland and one from Norway. Recent inventories have revealed a handful of new localities, but anyhow the species is very rare. All the collections were made in old, dry pine woodlands, where the basidiocarps of $\mathrm{H}$. gracilipes are found growing in the small space between long-ago fallen kelo trunk and forest soil. The fragile, rhizomorph-like stipe arises from the ground, and the pileus spreads along the wood surface above.

Phellodon secretus was described recently (Niemelä et al. 2003), almost exclusively from the materials of our inventories. This slender, pale ash-grey species resembles Phellodon connatus (Schultz : Fr.) P. Karst., but its spores are smaller and context hyphae make a soft and loose, interwoven structure, while $P$. connatus has densely packed and parallel contextual hyphae. The holotype of this species is one of our Koitajoki collections. Both this and H. gracilipes are surely threatened, but their Red List statuses in Finland have not yet been established.

Punctularia strigosozonata is a stereoid fungus, characterized by soft, small, cigar brown pilei and usually an effused-reflexed habit of the basidiocarps. Bondartseva et al. (2000) made a detailed overview on the species in Europe, where its distribution is clearly eastern, continental. Now this rare species has been found also in Finland: one record from our inventory area, plus two others nearby, close to the Mekrijärvi Biological Station of the University of Joensuu in Ilomantsi, and an old collection by TN from the Koli National Park in Lieksa. All these were found growing on Populus tremula. First Finnish finds were reported by Niemelä (2003b).

\subsection{Basidiocarp consistency classes}

It is no surprise that in the North Karelian Biosphere Reserve both the species composition of beetles and their spatial distribution inside polypore fruit bodies appeared characteristic for taiga zone in general. Up to $73 \%$ of fungivore beetles are known to be polyphagous (Schigel 2002).
They often show no preference to certain polypore genera or groups of related genera, but colonize polypores of certain consistency classes (Table 5).

This concept of basidiocarp consistency classes is here proposed to be used when describing different kinds of fungi as habitats for insects and their larvae. It is not yet fully understood, which particular characteristics of fungal basidiocarps are ecologically decisive to make them suitable for beetles. Critical are, for instance:

- Shape and volume of the fruit body;

- Annuality vs. perenniality, and how long the perennial ones persist;

- Presence or absence of certain structures, e.g. crust;

- Water contents of the basidiocarp;

- Toughness of the mycelium, which depends on the hyphal system (monomitic, dimitic, trimitic), thickness of hyphal walls, and how dense the structure is;

- Chemical characteristics of the fruit body.

The division of basidiocarp consistency classes, proposed here, is based on an informal classification long used by mycologists while describing species and genera. Consequently, the names of the consistency classes derive from certain polypore genera, but here taxonomy is omitted and the terms represent patterns of physical characteristics only. Basidiocarp consistency classes are outlined in Table 5.

Although these consistency classes seem to be valid and are repeatedly found in nature, sometimes it is difficult to define sharp borders between them: for instance, Ischnoderma resinosum Fr. (P. Karst.), a central European species, is tyromycetoid (= leptoporoid) when young but turns fomitoid when old (Pouzar 1971). Another example of uncertainty is found in the genus Trichaptum: T. abietinum, T. fuscoviolaceum and possibly $T$. laricinum (more data needed), all growing on coniferous trees, have a characteristic set of beetle species, unlike T. pargamenum on birch and other deciduous trees, which is usually colonized by specialists of the trametoid consistency class. Beetles of resupinate polypores are much less well known, and these fungi are more difficult to be addressed in certain consistency classes. Anyhow, some of them could well be placed in the same classes as the pileate ones, for instance Postia placenta and Sarcoporia salmonicolor among the tyromycetoid ones. 
Table 5. Basidiocarp consistency classes, and examples of characteristic polypore and beetle genera in European southern taiga.

\section{Fomitoid}

Hard, perennial, voluminous fruit bodies with thick context and several annual layers in hymenophore; robust when living; basidiocarps may stay attached on substrate for several years after death.

Examples of fungal genera: Fomes, Fomitopsis, Phellinus, Ganoderma, Heterobasidion.

Examples of beetles associated: Bolitophagus, Ennearthron, Dorcatoma, Oplocephala, Ropalodontus, Cis (subg. Eridaulus).

NB: In addition to beetle larvae adapted to develop in the hard context and trama, these fungi sometimes attract untypical imago, visiting sporulating fruit bodies (Latridiidae), or those covered by slime moulds (Leiodidae) or anamorphic fungi (Cryptophagidae). Larvae of beetles occur mostly in dying or dead fruit bodies. The life cycle may take more than one year.

\section{Trametoid}

Corky or leathery, projecting and fairly thin, shelf-shaped, fairly quickly drying, trimitic fruit bodies, annual.

Examples of fungal genera: Trametes, Daedaleopsis, Funalia, Lenzites, Gloeoporus, Pycnoporus, Cerrena, Bjerkandera.

Examples of beetles associated: Cis (C. comptus, C. hispidus, C. micans), Sulcacis, Octotemnus, Tritoma, Wagaicis.

NB: The tough context of dead fruit bodies is usually effectively eaten (generally by Ciidae) in dry condition the next season after sporulation. Larvae start to develop in living or dying fruit bodies. Several generations may utilize the cluster of fruit bodies before it is completely eaten.

\section{Tyromycetoid}

Soft and watery, monomitic, annual fruit bodies.

Examples of fungal genera: Tyromyces, Postia, Amylocystis, Leptoporus, Hapalopilus, Pycnoporellus.

Examples of beetles associated: Hallomenus.

NB: The high moisture contents of the fruit body and its short persistence limit the number of beetle species. Only two species of Hallomenus, pupating in soil, were found. Larvae eat living fruit bodies. Larval development is fast, one generation per year.

\section{Piptoporoid}

Corky or fleshy, voluminous, di/trimitic fruit bodies with thick and homogeneous context.

Examples of fungal genera: Piptoporus, Polyporus, Laetiporus.

Examples of beetles associated: Mycetophagus, Diaperis, Dacne, Eledona.

NB: Usually relatively large and thick fruit bodies, hosting both surface- and context-living larvae, which start to develop in either living or dying fruit bodies.

\section{Xanthochroic}

Brown coloured and monomitic, annual, at first fibrous, but becoming brittle upon dying.

Examples of fungal genera: Inonotus, Onnia.

Examples of beetles associated: Abdera, Orchesia, Mycetophagus.

NB: Larvae occupy the context of mostly living fruit bodies in somewhat similar way as the trametoid ones. Larvae very seldom pupate inside the fruit bodies, even if those usually stay on trunks for one or more years after their death. Beetles almost never colonise dead fruit bodies.

\section{Trichaptoid}

Thin, numerous, dimitic fruit bodies with purple coloured hymenophore; pilei merging at bases, annual, or continuing to grow over the next year.

Examples of fungal genera: Trichaptum (T. abietinum, T. fuscoviolaceum, ?T. laricinum).

Examples of beetles associated: Cis (C. punctulatus), Wanachia, Zilora.

NB: Beetle larvae settle at the confluent bases of the fruit bodies, where context thickness is sufficient to host larvae (Schigel 2002). 
Several polypore genera like Climacocystis, Gloeophyllum and Fistulina (Nikitsky \& Schigel 2004) have characteristic species assemblages and make consistency classes of their own. We see no sense to construct special names for them as far as there are no other genera sharing the same ecological characteristics.

\subsection{Families of fungivorous beetles}

Our study revealed a typical (Schigel 2002, Nikitsky \& Schigel 2004) palearctic set of beetle families that are linked to polypores, among which the Ciidae, Anobiidae (Dorcatoma), Melandryidae and Tenebrionidae are the most efficient basidiocarp destructors and decomposers. The Staphylinidae, Nitidulidae, Leiodidae, Trogossitidae, Latridiidae (Corticaria) and Corylophidae (Orthoperus) visit polypores as adult beetles. They feed on various parts of fruit bodies or secondary organisms, such as anamorphic fungi or slime moulds covering dead basidiocarps. The highest number of species were found among imaginal visitors, although the less diverse Ciidae, Anobiidae, Melandryidae and Tenebrionidae tend to be more abundant and were found either as larvae or were reared (Tables 2-3).

Fungivorous beetles of different families utilise fungal basidiocarps in different ways. Slime mould specialists in Leiodidae often visit polypores during the sporulation period together with Corylophidae and Latridiidae. The most diverse family, Staphylinidae, contains just a few proved fungivorous species, although many species recorded as imagines usually visit moist (both living and dead) polypores as well as agarics and boletes. Strongly decomposed and wet fruit bodies attract saprophagous imagines of the Silphidae, Hydrophilidae, Cholevidae, and Scarabaeidae. Anobiidae (Dorcatominae) larvae develop in the hardest polypores of the fomitoid consistency class. Trogossitidae larvae feed on wood-rotting mycelium, but imagines often stay on polypore hymenophore. Cryptophagidae use such anamorphic fungi that cover dead polypores. Erotylidae live on wood-rotting fungi and have rather short life cycles, which allows them to use ephemeral Pleurotus species and some short-persisting polypores (Inonotus obliquus, Trametes spp., Polyporus spp., Piptoporus betulinus). Ciidae live in various polypores which stay dry at least part of the decomposition time. Mycetophagidae,
Tetratomidae and Melandryidae larvae with short cycles occupy annual tyromycetoid and xanthochroic polypores. Each Tenebrionidae species colonizes a narrow set of polypore species, while the family as a whole has a fairly wide ecological amplitude, decomposing polypores of various consistency classes.

\subsection{The role of beetles as vectors of successor polypore species}

Niemelä at al. (1995) dealt with a very special link in which certain rare polypores share the woody substrate with a number of common poroid or hymenochaetoid fungi. In these cases a common and effective decayer inhabits a tree trunk. When its mycelium becomes senescent, another fungus species invades the tree, possibly killing the mycelium of the first species, and often fruiting on its dead basidiocarps. These predecessor-successor links may be fairly common in boreal forests, but little is known on their ecological background and even less on the mechanisms how the trees inhabited by a predecessor become inoculated by the mycelium of a successor.

By the time when these predecessor-successor relations were described, not much was understood about the spore dispersal of successor species, which are usually fairly rare and selective about their predecessors. We found that certain beetle larvae and successor polypores share similar preferences to the conditions of rotten wood they colonize. At the same time beetle imagines visit sporulating basidiocarps of both the predecessor and successor polypores, and hence spores of successors are present on the body of beetle female actively searching for an appropriate $\log$ to lay eggs.

Ostoma ferruginea is a beetle whose larvae live on wood brown-rotted by Fomitopsis pinicola and $F$. rosea, as well as on mycelia of certain successor polypores. Both imagines and larvae are often located in the transition between wood and fungus fruit body, and imagines feed on the hymenophore of Fomitopsis but also on secondary fungal species, which may colonize logs primarily decayed by Fomitopsis, e.g. Pycnoporellus fulgens and Antrodia albobrunnea. Basidiocarps of Pycnoporellus fulgens are often eaten by insect larvae fairly quickly after their development and beetles involved in the dispersal were expected to be found (J. Siitonen, pers. comm.). 
Ostoma ferruginea may further be involved in the spore dispersal of Amylocystis lapponica often growing together with Fomitopsis rosea on the same log. Imagines of $O$. ferruginea visit both of these polypore species. A. lapponica and $F$. rosea are known as co-existing species although no predecessor-successor relations were found on the basis of basidiocarp records. A. lapponi$c a$ fruit bodies never arise from the dead basidiocarps of $F$. rosea, and their parallel occurrence may be based on similar substrate preferences.

We believe that at least in some cases spores of successor polypores are dispersed both by wind and beetles, improving chances of rare successor polypores to maintain viable populations.

\subsection{Calculations}

Fresh basidiocarps attract the highest number of beetle species, collected as imagines (decomposition stage I, Figs. 5, 11). The decline in the number of species (imagines) during the decomposition of the fruit body is interrupted in stage III, when anamorphic fungi start to grow over the fruit body and attract specialized visitors, but structural changes and the decline of basidiocarp volume are not yet drastic. At the same time stages I and III are favoured by the larvae of fungivore beetles: Hallomenus and Abdera generally in stage I, while Dorcatoma and Ennearthron in stage III. These two peaks show that larvae of polypore-dwelling beetles use a strategy either of a short life cycle starting in living fruit body, or a more long one in recently dead polypore basidiocarp. Cis larvae and imagines were found in all the stages (Figs. 5-8, 11).

\subsection{Notes on selected beetles}

Of the two Leiodidae species found, only Agathidium pisanum was observed feeding on the hymenophore surface of the polypore Rigidoporus corticola. A. arcticum imagines were found on Reticularia sp., a slime mould.

Imagines of Phloeocharis subtilissima hide inside the tubes or wander on the surface of the hymenophore of Phellinus pini. Vibration of substrate makes beetles escape in the tubes, where they possibly consume spores. Similar behaviour was shown for another staphylinoid (Ptiliidae) beetle Baranowskiella ehnstromi Sörensson, 1997 in the much more fine tubes of Phellinus conchatus (Sörensson 1997).

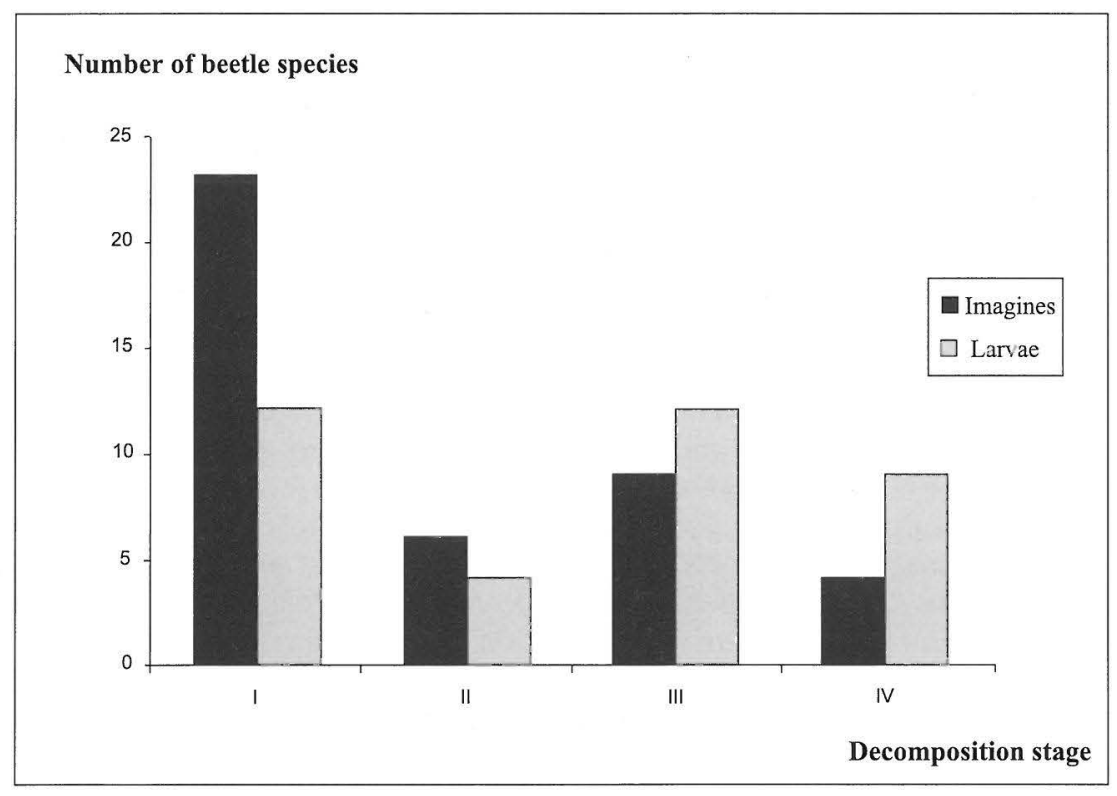

Fig. 11. Numbers of beetle species during the decomposition of fungal basidiocarps. The study was made with polypores of the North Karelian Biosphere Reserve, Finland. For the decomposition stages (I-IV) see Table 1. 
Imagines of Atheta boleticola were found on the hymenophore of Phaeolus schweinitzii together with numerous unidentified staphylinoid larvae living inside the spongy context. Rearings failed, but it is possible that larvae of $A$. boleticola feed on this polypore. Most of Staphylinidae were found on the hymenophore of various polypores, usually moistened by wet soil, dew, or atmospheric precipitation. However, the larval host preferences of the Staphylinidae would deserve further study.

Two related $C i$ s species are known to develop in basidiocarp context and on the transition layer between hymenophore and context (Schigel 2002), which was supported in our study with other substrates: Cis comptus in Amylocystis lapponica and Gloeophyllum sepiarium, and Cis hispidus in Antrodia pulvinascens, Ceriporiopsis resinascens and Lenzites betulinus. In Trametes it was found that species composition of the Ciidae includes three size classes of beetle larvae (and emerging adults) interlacing their burrows of different diameters simultaneously, as it was found in European Russia (Schigel 2002). These three classes in the North Karelian Nature Reserve are represented by Cis boleti (largest burrows), C. hispidus (medium-sized) and Octotemnus glabriculus (narrowest), respectively. Burrows of the larvae merge in the late stages of the fruit body decomposition. Fruit body size limits the spatial distribution of larvae inside the basidiocarp: large larvae of Cis boleti are located in fruit bodies of Trametes ochracea, T. pubescens and $T$. velutina at their thick base (umbo), while larvae of Cis hispidus and, in particular, Octotemnus glabriculus tend to graze inside the inner parts of the basidiocarp margin.

Imagines and larvae of Cis dentatus burrow the resupinate but fairly thick fruit bodies of Sarcoporia salmonicolor and aggregate in their dryer and thicker parts. C. punctulatus larvae develop usually in Trichaptum fuscoviolaceum and T. abietinum (Kompantsev 1982, Schigel 2002) but we reared a few individuals also from $T$. pargamenum, which is typically colonized by other ciids.

Dolichocis laricinus larvae develop in the strongly decomposed fruit bodies of Antrodia pulvinascens and Polyporus leptocephalus (P. varius).

Imagines of Rhizophagus dispar, Acrulia inflata and Orthoperus atomus were found on the surface of Phellinus laevigatus around excrements of unidentified Tineidae larvae. Imagines of $R$. dispar on the fruit bodies of Gelatoporia pannocincta were usually found on excrements of Diptera and Lepidoptera larvae. Processed fungal substrate is more attractive to certain beetle generalists than the hard hyphae of an intact hymenophore. In particular imagines of Rhizophagus tend to occur on sporulating polypores (Inonotus obliquus and Fomitopsis pinicola) but decompose also other fungal substrates, especially euagarics (Moncalvo et al. 2002) such as Pleurotus species and Hypsizygus ulmarius. Rhizophagus was found both on basidiocarps on standing trees and on fruit bodies fallen on the ground. Generally, unlike polypores, euagarics attract less beetles from families other than Stapylinidae, although in Europe the beetle fauna of these two large groups of fungi partly overlap.

Colonization of the fruit bodies of Phellinus (P. pini, P. populicola, $P$. igniarius complex) usually starts when the area of living hymenophore starts to shrink. From this moment the upper part of a fruit body begins to die off. This stage of decomposition (III) is preferred by Dorcatoma larvae, for instance Dorcatoma dresdensis in Phellinus lundellii. Fruit bodies of $P$. laevigatus are sometimes quite thin, less than $1 \mathrm{~cm}$, and in this case the sickle-shaped larvae of Dorcatoma change their typical vertical position to horizontal, but still avoid the hymenophore. In dead fruit bodies larvae can consume also the hymenophore. During the whole development cycle the larvae of Dorcatoma tend to avoid the outer 1-cm zone of the fruit body. This avoided distance is greater still in the fruit bodies of $P$. populicola because of the deep cracks of the upper side of the fungus. On the one hand, avoiding outer layers of the substrate is characteristic for nearly all the substrate dwellers because of the lower risk of parasite attack and more stable substrate conditions deeper in, but on the other hand imagines emerging from pupae and leaving the fungus face certain difficulties to come out. However, Anobiidae are adapted to live in hard and dry substrates, and Doracatominae occupy the niche of decomposers of robust polypores. They can also appear at the early stages of fruit body decomposition, thus avoiding competition with Ciidae and other Coleoptera.

Imagines and larvae of Ennearthron cornutum are located between the living hymenophore of Phellinus pini and the half-dead context of this long-persisting fungus, or in senescent inner layers of the hymenophore. Similar distribu- 
tion was found in P. conchatus and P. chrysolo$m a$, where larvae are using mostly the marginal parts of the fruit body. $P$. viticola is softer in its consistency, and harbours larvae close to the context. On the contrary, Dichomitus squalens is a soft polypore, and Ennearthron cornutum larvae are restricted to the context. E. cornutum is a generalist beetle, but the preferred parts of fruit bodies vary according to fungus species.

The more firm and structured the polypore fruit body is, the more complex the structure of spatial distribution of larvae inside the fungal substrate tends to be. Soft and homogeneous context will lead in an even distribution of larvae: Ennearthron cornutum in Dichomitus squalens; larvae of Sulcacis affinis in the context of Pyconoporus cinnabarinus; Hallomenus sp. larvae in Postia alni, $P$. fragilis, $P$. lateritia, $P$. leucomallella, $P$. placenta, P. stiptica, P. tephroleuca and Amylocystis lapponica.

Dromius sigma, a small, common carabid species, is an inhabitant of lake- and riversides, living under leaves of Alnus and Salix. Sometimes occasional individuals move far from water in untypical habitats, like the polypore Cerrena unicolor.

Acknowledgements: Parts of the Coleoptera material were identified by Dr. N.B. Nikitsky (Zoological Museum of M.V. Lomonosov Moscow State University, Russia), Mr. A.V. Kompantsev and Mrs. T.V. Kompantseva (A.N. Severtsov Institute of Ecology and Evolution, Moscow, Russia), and Mr. V.B. Semenov (E. I. Marcinovsky Institute of Medical Parasitology and Tropical Medicine). We are grateful for this assistance. We appreciate the valuable comments of Dr. Hans Silfverberg (Finnish Museum of Natural History). The Finnish Forest and Park Service kindly invited us to carry out this study and provided facilities for the field work. E-mail discussions in the Finnish colepterist mailing list "Kuoriaisposti" helped to solve a problem in beetle synonymy. A research grant from the Ministry of Environment, Finland (YM131/5512/2002) is gratefully acknowledged.

\section{References}

Ahti, T., Hämet-Ahti, L. \& Jalas, J. 1968: Vegetation zones and their sections in northwestern Europe. Ann. Bot. Fennici 5:169-211.

Andersen, J., Olberg, S. \& Haugen, L. 2000: Saproxylic beetles (Coleoptera) of Troms and Western Finnmark, northern Norway with exceptional distribution in Fennoscandia. - Norwegian J. Entomol. 47: 29-40.

Benick, L. 1952: Pilzkäfer und Käferpilze. Ökologische und statistische Untersuchungen. - Acta Zool. Fennica 70: 1-309.
Bondarceva, M.A., Krutov, V.I., Lositskaya, V.M. \& Svishch, L.G. 1995: Aphyllophoraceous fungi in some pine forests in Eastern Finland and Russian Karelia. In: Hokkanen, T. J. \& Leshko, I. (eds.), Karelian Biosphere Reserve Studies. North Karelian Biosphere Reserve, Joensuu: 195-201.

Bondartseva, M.A., Lositskaya, V.M. \& Hokkanen, T.J. 1999: Aphyllophoroid fungi of the North Karelian Biosphere Reserve (Finland). - Kew Bull. 54: 589 603.

Bondartseva, M., Lositskaya, V., Hokkanen, T. \& Krutov, V. 2001: Studies on Aphyllophoraceous fungi in North Karelian Biosphere Reserve 1993-1996. Nature Protection Publ. Finnish Forest and Park Service A 131: 91-111.

Bondartseva, M.A., Lositskaya, V.M. \& Zmitrovich, I.V. 1998: Aphyllophoroid fungi of old and primeval forests in the Kotavaara site of North Karelian Biosphere Reserve. - Folia Cryptog. Estonica 33: 19-24.

Bondartseva, M.A., Lositskaya, V.M. \& Zmitrovich, I.V. 2000: Punctularia strigosozonata (Punctulariaceae) in Europe. - Karstenia 40: 9-10.

Donisthorpe, H. 1931: Coleoptera found in the "birch bracket" fungus Polyporus betulinus. - Entomol. Res. 43: 14 .

Donisthorpe, H. 1935: The British fungicolous Coleoptera. - Entomol. Monthly Mag. 71: 21-31.

Ehnström, B. \& Axelsson, R. 2002: Insektsgnag i bark och ved. - ArtDatabanken SLU: 165-498.

Hansen, L. \& Knudsen, H. 1997: Nordic Macromycetes 3. Heterobasidioid, aphyllophoroid and gastromycetoid Basidiomycetes. - Nordsvamp, Copenhagen. 444 pp.

Hokkanen, T.J. \& Ieshko, E. 1995: Karelian Biosphere Reserve studies. - North Karelian Biosphere Reserve, Joensuu. 278 pp.

Jonsell, M. \& Norlander, G. 1995: Field attraction of Coleoptera to odours of the wood-decaying polypores Fomitopsis pinicola and Fomes fomentarius. - Ann. Zool. Fennici 32: 391-402.

Jonsell, M. \& Nordlander, G. 2002: Insects in polypore fungi as indicator species: a comparison between forest sites differing in amounts and continuity of dead wood. - Forest Ecol. Managem. 157: 101-118.

Kõljalg, U. \& Renvall, P. 2000: Hydnellum gracilipes, a link between stipitate and resupinate Hymenomycetes. - Karstenia 40:71-77.

Komonen, A., Siitonen, J. \& Mutanen, M. 2001: Insects inhabiting two old growth forest polypore species. Entomol. Fennica 12: 1-14.

Kompantsev, A.V. 1982: (Morpho-ecological peculiarities of Erotylidae (Coleoptera) larvae - inhabitants of the fruit bodies of higher fungi). - In: Morphoecological adaptations of insects in terrestrial communities: 81-91. Nauka, Moskva. (In Russian.)

Kotiranta, H. \& Niemelä, T. 1996: Uhanalaiset käävät Suomessa. 2nd revised edition. - Edita, Helsinki. 184 pp.

Lawrence, J. F. 1973: Host preference in Ciid beetles (Coleopetra: Ciidae) inhabiting the fruiting bodies of Basidiomycetes in North America. - Bull. Mus. Comparative Zool. 145: 163-212.

Martikainen, P. 2001: Conservation of threatened saproxylic beetles: significance of retained aspen Populus tremula on clearcut areas. - Ecol. Bull. 49: 205-218. 
Moncalvo, J.M., Vilgalys, R., Redhead, S.A., Johnson, J.E., James, T.Y., Aime, M.C., Hofstetter, V., Verduin, S.J.W., Larsson, E., Baroni, T.J., Thorn, R.G., Jacobsson, S., Clémençon, H. \& Miller, O.K. Jr. 2002: One hundred and seventeen clades of euagarics. - Molecular Phylogen. Evolution 23: 357-400.

Müller, C., Jaeger, B., Kompantsev, A.V. \& Uhlig, M. 2001: Type and species catalogue of the minute treefungus beetles of the Museum für Naturkunde in Berlin, with general information on the Coleoptera collection, its curation and "Historical collection" (Coleoptera, Polyphaga, Ciidae and Pterogeniidae). - Mitt. Mus. Naturkund. Berlin, Zool. Reihe 77: 303-323.

Niemelä, T. 1980: On Fennoscandian polypores 7. The genus Pycnoporellus. - Karstenia 20:1-15.

Niemelä, T. 2003a: What is the commonest polypore in Finland? - Sienilehti 55: 46-53. (In Finnish.)

Niemelä, T. 2003b: Collecting Punctularia strigosozonata (Corticiaceae) in Finland and Estonia. - Sienilehti 55: 67-70. (In Finnish.)

Niemelä, T. 2004: Guide to the polypores of Finland. 15 th revised edition. - Bot. Bull. Univ. Helsinki 184: $1-148$.

Niemelä, T. \& Dai, Y.C. 1998: Luoston suojelualueen kääpäinventointi 1998. - Metsähallitus, Perä-Pohjolan luontopalvelut, Helsinki. 32 pp.

Niemelä, T. \& Dai, Y.C. 1999: Polypores of Luosto. Nature Protection Publ. Finnish Forest Park Service A 105: 1-59.

Niemelä, T. \& Kinnunen, J. 2001: Korouoman suojelualueen kääpäinventointi 2001. - Metsähallitus, PeräPohjolan luontopalvelut, Helsinki. 38 pp.

Niemelä, T. \& Kinnunen, J. 2002: Ylläksen-Aakenuksen suojelualueen kääpäinventointi 2001. - Metsähallitus, Perä-Pohjolan luontopalvelut, Helsinki. 30 pp.

Niemelä, T. \& Kinnunen, J. 2003: Pisavaaran luonnonpuiston kääpäinventointi 2003. - Metsähallitus, PeräPohjolan luontopalvelut, Helsinki. 16 pp.

Niemelä, T., Kinnunen, J. \& Manninen, O. 2002: Koitajoen Natura-alueen kääpäinventointi 2002. - Metsähallitus, Itä-Suomen luontopalvelut, Helsinki. 41 pp.

Niemelä, T., Kinnunen, J., Renvall, P. \& Schigel, D. 2003: Phellodon secretus (Basidiomycota), a new hydnaceous fungus from northern pine woodlands. Karstenia 43: 37-44.

Niemelä, T., Renvall P., Penttilä R. 1995: Interactions of fungi at late stages of wood decomposition. - Ann. Bot. Fennici 32: 141-152.

Nikitsky, N.B. \& Schigel, D.S. 2004: Beetles in polypores of the Moscow region: checklist and ecological notes. - Entomol. Fennica 15: 6-22.

Økland, B. 1995: Insect fauna compared between six polypore species in a southern Norwegian spruce forest. - Fauna Norvegica B 42: 21-46.
Palm, T. 1951: Die Holz- und Rindenkäfer der nordschwedische Laubbäume. - Meddel. Statens Skogsforskningsinst. 40: 1-242.

Palm, T. 1959: Die Holz- und Rindenkäfer der süd- und mittelschwedischen Laubbäume. - Opusc. Entomologica Suppl. 16: 1-374.

Pavior-Smith, K. 1960: The fruiting bodies of macrofungi as habitats for beetles of the family Ciidae (Coleoptera). - Oikos 11: 1-17.

Pouzar, Z. 1971: Notes on taxonomy and nomenclature of Ischnoderma resinosum (Fr.) P. Karst. and I. benzoinum (Wahlenb.) P. Karst. (Polyporaceae). - Česká Mykologie 25: 15-21.

Rassi, P., Alanen, A., Kanerva, T. \& Mannerkoski, I. (eds.) 2001: The 2000 Red List of Finnish species. Ministry of the Environment \& Finnish Environment Institute: 1-432.

Saalas, U. 1917: Die Fichtenkäfer Finnlands. - Ann. Acad. Sci. Fenniae A 8: 1-547.

Saalas, U. 1923: Die Fichtenkäfer Finnlands. - Ann. Acad. Sci. Fenniae B 22: 1-746.

Schigel, D.S. 2002: (Beetle complexes in polypore fungi in East European Plain and Crimea). - Bull. Moscow Soc. Naturalists 107: 8-21. (In Russian.)

Shubin, V.I. \& Krutov, V.I. 1979: Griby Karelii i Murmanskoy oblasti. - Nauka, Leningrad. 104 pp.

Siitonen, J., Penttilä, R. \& Kotiranta, H. 2001: Coarse woody debris, polyporous fungi and saproxylic insects in an old-growth forest in Voldozero National Park, Russian Karelia. - Ecological Bulletins 49: 231-242.

Silfverberg, H. 1992: Enumeratio Coleopterorum Fennoscandiae, Daniae et Baltiae. - Helsingin Hyönteisvaihtoyhdistys, Helsinki. 94 pp.

Sörensson, M. 1997: Morphological and taxonomical novelties in the world's smallest beetles, and the first Old World record of Nanosellini (Coleoptera: Ptiliidae). - Systematic Entomol. 22: 257-283.

Thunes, K.H. 1994: The coleopteran fauna of Piptoporus betulinus and Fomes fomentarius (Aphyllophorales: Polyporaceae) in western Norway. - Entomol. Fennica 5: $157-168$.

Thunes, K.H., Midtgaard, F. \& Gjerde, I. 2000: Diversity of coleoptera of the bracket fungus Fomitopsis pinicola in a Norwegian spruce forest. - Biodiversity and Conservation 9: 833-852.

Yakovlev, E. B., Nikitsky, N. B. \& Sherbakov, A. 2001: Saproxylic Coleoptera of unmanaged mature forests in Koitajoki area. - Diversity studies in Koitajoki Area (North Karelian Biosphere Reserve, Ilomantsi, Finland). Nature Protection Publ. Finnish Forest Park Service A 131: 32-71. 